Linköping Studies in Science and Technology, Licentiate Thesis No. 1612

\title{
A class of infinite dimensional stochastic processes with unbounded diffusion
}

\author{
John Karlsson
}

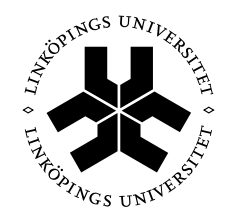

Linköping University INSTITUTE OF TECHNOLOGY

Department of Mathematics

Linköping University, SE-581 83 Linköping, Sweden

Linköping 2013 
Linköping Studies in Science and Technology, Licentiate Thesis No. 1612

\title{
A class of infinite dimensional stochastic processes with unbounded diffusion
}

John Karlsson

\author{
john.karlssondiu.se \\ www.mai.liu.se \\ Mathematical Statistics \\ Department of Mathematics \\ Linköping University \\ SE-581 83 Linköping \\ Sweden
}

\section{LIU-TEK-LIC-2013:46 \\ ISBN 978-91-7519-536-0 \\ ISSN 0280-7971}

Copyright (c) 2013 John Karlsson

Printed by LiU-Tryck, Linköping, Sweden 2013, 2nd printing

This is a Swedish Licentiate Thesis. The Licentiate degree comprises 120 ECTS credits of postgraduate studies. 




\section{Abstract}

The aim of this work is to provide an introduction into the theory of infinite dimensional stochastic processes. The thesis contains the paper A class of infinite dimensional stochastic processes with unbounded diffusion written at Linköping University during 2012. The aim of that paper is to take results from the finite dimensional theory into the infinite dimensional case. This is done via the means of a coordinate representation. It is shown that for a certain kind of Dirichlet form with unbounded diffusion, we have properties such as closability, quasi-regularity, and existence of local first and second moment of the associated process. The starting chapters of this thesis contain the prerequisite theory for understanding the paper. It is my hope that any reader unfamiliar with the subject will find this thesis useful, as an introduction to the field of infinite dimensional processes. 



\section{Populärvetenskaplig sammanfattning}

En stokastisk process är något som beskriver hur ett slumpmässigt system ändrar sig över tiden, t.ex. hur antalet kunder i en affär ändras under dagen eller hur värdet av en aktie förändras. Stokastiska processer har studerats i över 100 år och det finns många olika typer av dem. En av de enklaste typerna är Markovprocessen som är en slumpmässig process med "dåligt minne". Nästa värde som processen har beror bara på dess nuvarande tillstånd. Markovprocesser har studerats sedan början av 1900-talet när A. A. Markov studerade hur konsonanter och vokaler följer efter varandra i texter. En Markovprocess i kontinuerlig tid (d.v.s. inga hopp) som följer en kontinuerlig kurva kallas för en diffusionsprocess. En process kan ta värden i flera dimensioner, exempelvis kan en partikels bana i rummet sägas vara tredimensionell. Den här avhandlingen behandlar oändligdimensionella processer d.v.s. processer som tar värden i rum med oändlig dimension. Man har visat att en viss typ av matematiskt objekt, en så kallad Dirichletform, ibland beskriver en sådan process. Den här avhandlingen studerar en speciell sorts Dirichletform och hittar krav för att vi ska kunna associera den med en process. Det är möjligt att dessa oändligdimensionella processer har viktig tillämpning inom teoretisk fysik. 



\section{Contents}

1 Introduction 1

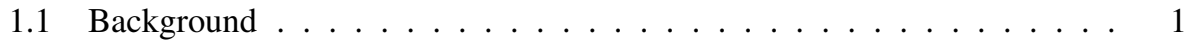

1.2 Outline ..................... 2

2 Chapter 2-Malliavin Calculus 5

2.1 General framework . . . . . . . . . . . . . . . . 5

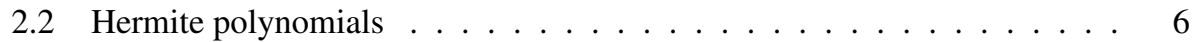

2.3 Wiener Chaos . . . . . . . . . . . . . . . . . . . 6

2.4 Multiple stochastic integrals $\ldots \ldots \ldots \ldots \ldots$

2.5 Derivative operator . . . . . . . . . . . . . . . . . 11

2.6 Divergence operator . . . . . . . . . . . . . . . . . 15

2.7 The generator of the Ornstein-Uhlenbeck semigroup $\ldots \ldots \ldots$

3 Chapter 3-Dirichlet form theory 21

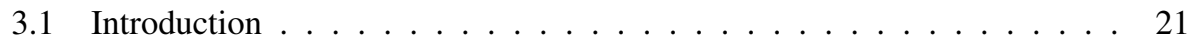

3.2 Resolvents . . . . . . . . . . . . . . . . . . . . . . 21

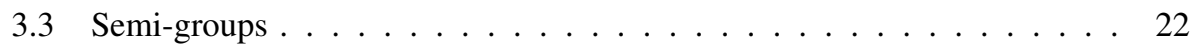

3.4 Bilinear forms . . . . . . . . . . . . . . . . . . . . 23

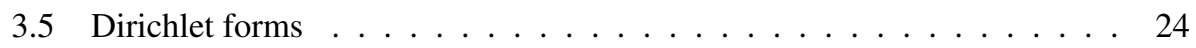

4 Chapter 4-Main results $\quad 29$

4.1 Earlier work . . . . . . . . . . . . . . . . . . . . . . . 29

4.2 Introduction . . . . . . . . . . . . . . . . . 31

4.3 Formal definitions . . . . . . . . . . . . . . . . . 32

4.4 Definition of the form . . . . . . . . . . . . . . . . 33

4.5 Closability . . . . . . . . . . . . . . . . . . 33

4.6 Quasi-regularity . . . . . . . . . . . . . . . . . . . 39 
4.7 The generator . . . . . . . . . . . . . . . . . . 41

4.8 Local first moment . . . . . . . . . . . . . . . . . . . 43

5 Chapter 5-Notation and basic definitions 47

5.1 Introduction . . . . . . . . . . . . . . . . . 47

5.2 Definitions . . . . . . . . . . . . . . . . . . 47

5.3 Notation . . . . . . . . . . . . . . . . . . . . . 48

Bibliography 


\section{Introduction}

$\mathrm{T}$ HE aim of this work is to provide an introduction into the theory of infinite dimensional stochastic processes. The thesis contains the paper A class of infinite dimensional stochastic processes with unbounded diffusion, a joint work with Jörg-Uwe Löbus, written at Linköping University during 2012. The starting chapters of the thesis contain the prerequisite theory for understanding the paper and it is my hope that any reader unfamiliar with the subject will find this useful as an introduction to the field.

\subsection{Background}

The theory of stochastic processes goes back to the beginning of the 20th century, and in the middle of the 20th century many important concepts were formed. An excellent overview of this is the article of P.-A. Meyer [6]. Doob worked on Martingales in the time period 1940-1950 and in 1945 a paper was published detailing the strong Markov property. Itô had his first article about the stochastic integral published in 1944 which clearly has had a large impact on current theory. In 1948 Hille and Yoshida formulated the theorem describing the semigroups of continuous operators. This had a large impact on Markov theory since the nature of a Markov process provides a natural connection to semigroups. Markov processes are of interest due to their natural occurence. As mentioned, because the evolution of a Markov process only depends on its present state, any observation in nature could be considered Markov provided there is sufficient information. If the time is discrete we call it a Markov chain. While Markov chains change value at discrete times a diffusion is a Markov process with continuous trajectories. From Kolmogorov we know that for a Markov process, the transition function is usually a solution of the Focker-Planck parabolic partial differential equation. In 1959 Beurling and Deny introduced the theory of Dirichlet forms. Their paper was in the area of potential theory. This theory was brought into the realm of probablity by Silverstein in 1974 and Fukushima in 1975. This is a useful tool for creating Markov processes in infinite dimen- 
sional spaces, something that might have applications in physics. In 1976 P. Malliavin introduced the theory of Malliavin calculus, first developed to prove a theorem in the theory of partial differential equations. From the Malliavin calculus comes the Wiener chaos and Ornstein-Uhlenbeck operator which can be used for analysis on the Wiener space and thus allowing analysis on infinite dimensions, once again with possible applications in theoretical physics.

In 1992 B. Driver and M. Röckner [2] published a paper detailing the construction of an "Ornstein-Uhlenbeck" like process on the path space of a compact Riemannian manifold without boundary.

In 2004 J.-U. Löbus published a paper [4] describing a class of processes on the path space of a Riemannian manifold. In this paper the operator describing the diffusion was unbounded in contrast to earlier papers.

F.-Y. Wang and B. Wu generalized the results in these papers and showed that the Riemannian manifold can be noncompact, in addition they extended the class of Dirichlet forms studied. Their paper [11] was published in 2008. The same authors published another paper [12] in 2009 detailing a large class of quasi-regular Dirichlet forms with unbounded, and non-constant diffusion coefficients on free Riemannian path spaces.

\subsection{Outline}

This thesis consists of five chapters and the outline is as follows.

\section{Chapter 1}

This chapter contains a historical overview of the theory. It also contains the purpose of this work as well as providing an outline of the layout of the thesis.

\section{Chapter 2}

Chapter two presents the basics of Malliavin Calculus. The chapter begins with introducing the Hermite polynomials as a starting point for the Wiener chaos decomposition. It is then shown that this decomposition has a representation in the form of Itô integrals. We then present the definition of the Malliavin gradient $D$ and some of its properties, also connecting it to the Wiener chaos decomposition. The end of the chapter contains a presentation of the divergence operator $\delta$, the generator of the Ornstein-Uhlenbeck semigroup $L$, and the relationship between $D, \delta$ and $L$.

\section{Chapter 3}

Chapter three is an introduction to the theory of Dirichlet forms. The first part of the chapter recalls the definitions of resolvents, semigroups, and the infinitesimal generator associated to them. We also state the famous Hille-Yosida theorem for contraction semigroups and by that note the connection between semigroup, resolvent, and generator. The second part of the chapter connects the first part to bilinear forms. The last part of the chapter states the definition of a Dirichlet form and quasi-continuity. We also state the 
theorem connecting the Dirichlet form to a stochastic process. The section ends with stating the famous Cameron-Martin theorem.

\section{Chapter 4}

Chapter four contains the paper A class of infinite dimensional stochastic processes with unbounded diffusion written at Linköping University during 2012. The version here is more detailed than the preprint version at ArXiv. The paper is a study of a particular kind of Dirichlet form. We study the form on cylindrical functions and under some assumptions we prove the closability of the form. The form is shown to be quasi-regular. The last parts of the paper studies the local first and second moment of the associated process.

\section{Chapter 5}

Chapter five contains elementary definitions of concepts used in the thesis. The section also contains a list of notation and abbreviations used throughout this work. 



\section{2}

\section{Chapter 2-Malliavin Calculus}

7 HE Malliavin calculus, named after the French matematician Paul Malliavin, extends

1 the calculus of variations, from functions to stochastic processes. In particular it makes it possible to calculate the derivative of random variables. This section serves to introduce the notions used in the field of Malliavin calculus. The section is heavily based on David Nualart's monographs [8] and [7].

\subsection{General framework}

Let $\mathbb{H}$ be a real separable Hilbert space with inner product $\langle\cdot, \cdot\rangle_{\mathbb{H}}$ and corresponding norm $\|\cdot\|_{\mathbb{H}}$. In this presentation the Hilbert space $\mathbb{H}$ will mostly be considered to be $L^{2}([0,1])$ with the Borel $\sigma$-algebra $\mathcal{B}$ and Lebesgue measure $\mu$, while in a more general setting it would be $L^{2}(T, \mathcal{B}, \mu)$ where $(T, \mathcal{B})$ is a measurable space and $\mu$ is an atomless measure on $(T, \mathcal{B})$.

Definition 2.1.1. A stochastic process $W=\{W(h), h \in \mathbb{H}\}$ defined in a complete probability space $(\Omega, \mathcal{F}, P)$ is a Gaussian process on $\mathbb{H}$, if $W$ is a centered Gaussian family of random variables and $E[W(g) W(h)]=\langle g, h\rangle_{\mathbb{H}}$ for all $g, h \in \mathbb{H}$.

This is illustrated by a simple example.

Example 2.1

Let $\mathbb{H}=L^{2}(\mathbb{R}+)$ and $W_{t}:=W(\mathbb{1}[0, t]), t \geq 0$. This is standard Brownian motion since

$$
E\left[W_{s} W_{t}\right]=E\left[W\left(\mathbb{1}_{[0, s]}\right) W\left(\mathbb{1}_{[0, t]}\right)\right]=\left\langle\mathbb{1}_{[0, s]}, \mathbb{1}_{[0, t]}\right\rangle_{L^{2}}=\min (s, t) .
$$

Using the same notation as in Nualart [8] letting $\mathcal{H}_{1}:=W$, we note that $\mathcal{H}_{1}$ is a closed 
subspace of $L^{2}(\Omega, \mathcal{F}, P)$, and that $W: \mathbb{H} \rightarrow \mathcal{H}_{1}$ defines a linear isometry between $\mathbb{H}$ and $\mathcal{H}_{1}$. We will denote by $W(A):=W\left(\mathbb{1}_{A}\right)$. Given a Hilbert space $\mathbb{H}$, a probability space and Gaussian process satisfying the above conditions exist. This is a consequence of Kolmogorov's extension theorem.

\subsection{Hermite polynomials}

The Hermite polynomials will play an important role in the upcoming Wiener chaos decomposition of a Gaussian random variable. The Hermite polynomials $H_{n}$ are the coefficients of the Taylor expansion of $F(x, t)=\exp \left\{t x-t^{2} / 2\right\}$ in powers of $t$, that is:

$$
F(x, t):=e^{t x-\frac{t^{2}}{2}}=\sum_{n=0}^{\infty} H_{n}(x) \cdot t^{n} .
$$

We have the relations:

$$
H_{0}(x)=1, \quad H_{1}(x)=x, \quad H_{2}(x)=\frac{1}{2}\left(x^{2}-1\right), \quad H_{n}(x)=\frac{(-1)^{n}}{n !} e^{\frac{x^{2}}{2}} \frac{d^{n}}{d x^{n}}\left(e^{-\frac{x^{2}}{2}}\right)
$$

and also:

$$
\frac{d}{d x} H_{n}(x)=H_{n-1}(x), \quad(n+1) H_{n+1}(x)=x H_{n}(x)-H_{n-1}(x)
$$

as well as:

$$
H_{n}(0)= \begin{cases}0 & \text { if } n \text { odd } \\ \frac{(-1)^{k}}{2^{k} k !} & \text { if } n \text { even. }\end{cases}
$$

Remark 2.2.1. The term Hermite polynomial does not have a strict definition. In other literature the polynomials $\tilde{H}$ or $\hat{H}$ defined by

$$
e^{t x-\frac{t^{2}}{2}}=\sum_{n=0}^{\infty} \tilde{H}_{n}(x) \cdot \frac{t^{n}}{n !}
$$

or

$$
e^{2 t x-\frac{t^{2}}{2}}=\sum_{n=0}^{\infty} \hat{H}_{n}(x) \cdot \frac{t^{n}}{n !}
$$

are classifed as the Hermite polynomials.

\subsection{Wiener Chaos}

Assume that $\mathbb{H}$ (we may think of it as $L^{2}([0,1])$ ) is infinite dimensional and let $\left\{e_{1}, e_{2}, \ldots\right\}$ be an ON-basis in $\mathbb{H}$. We let $\Lambda$ denote the set of all finite multi-indices $a=\left(a_{1}, a_{2}, \ldots\right)$, $a_{i} \in \mathbb{N}$ for $i=1,2, \ldots$, i.e. only finitely many $a_{i}$ :s are non-zero. For any $a \in \Lambda$ we define:

$$
a !:=\prod_{i=1}^{\infty} a_{i} !, \quad|a|:=\sum_{i=1}^{\infty} a_{i}, \quad \Phi_{a}:=\sqrt{a !} \prod_{i=1}^{\infty} H_{a_{i}}\left(W\left(e_{i}\right)\right) .
$$

Gaussian variables and Hermite polynomials are connected. The next lemma is related to orthogonality in $L^{2}(\Omega, \mathcal{F}, P)$. 
Lemma 2.3.1. If $(X, Y)$ is a two-dimensional Gaussian vector s.t. $E[X]=E[Y]=0$ and $V[X]=V[Y]=1$. Then for all $m, n \in \mathbb{N}$ we have:

$$
E\left[H_{m}(X) H_{n}(Y)\right]= \begin{cases}0 & \text { if } m \neq n, \\ \frac{1}{n !} E[X Y]^{n} & \text { if } m=n .\end{cases}
$$

Proof: For a Gaussian random variable $Z$ we have $E\left[e^{Z}\right]=e^{\frac{1}{2} E\left[Z^{2}\right]}$ and thus

$$
\begin{aligned}
E\left[e^{s X+t Y}\right] & =e^{\frac{1}{2} E\left[(s X+t Y)^{2}\right]}=e^{\frac{1}{2} E\left[s^{2} X^{2}\right]} e^{\frac{1}{2} E\left[t^{2} Y^{2}\right]} e^{E[s t X Y]} \\
& =e^{\frac{1}{2} s^{2}} e^{\frac{1}{2} t^{2}} e^{E[s t X Y]},
\end{aligned}
$$

and we get

$$
e^{s t E[X Y]}=E\left[e^{s X-\frac{s^{2}}{2}} e^{t Y-\frac{t^{2}}{2}}\right]=E\left[\left(\sum_{m=0}^{\infty} s^{m}\left(H_{m}(X)\right)\left(\sum_{n=0}^{\infty} t^{n} H_{n}(Y)\right)\right] .\right.
$$

Taylor expansion of the left hand side gives:

$$
e^{s t E[X Y]}=1+s t E[X Y]+\frac{s^{2} t^{2} E[X Y]^{2}}{2 !}+\cdots+\frac{s^{n} t^{n} E[X Y]^{2}}{n !}+\ldots
$$

and identifying coefficients of $s^{m} t^{n}$ yields:

$$
E\left[H_{m}(X) H_{n}(Y)\right]= \begin{cases}0 & \text { if } m \neq n \\ \frac{1}{n !} E[X Y]^{n} & \text { if } m=n .\end{cases}
$$

Using this lemma we can proceed to prove that the Hermite polynomials can be used to construct an $\mathrm{ON}$-basis for $L^{2}(\Omega, \mathcal{F}, P)$.

Proposition 2.3.2. The collection $\left\{\Phi_{a}: a \in \Lambda\right\}$ is an ON-basis for $L^{2}(\Omega, \mathcal{F}, P)$.

Proof: Step 1: We begin by showing that $\left\{\Phi_{a}: a \in \Lambda\right\}$ forms an orthonormal system. Let $a, b \in \Lambda$. Since $W\left(e_{i}\right)$ and $W\left(e_{j}\right)$ are independent for $i \neq j$ we get:

$$
\begin{aligned}
E\left[\Phi_{a} \Phi_{b}\right] & =E\left[\sqrt{a !} \prod_{i=1}^{\infty} H_{a_{i}}\left(W\left(e_{i}\right)\right) \cdot \sqrt{a !} \prod_{i=1}^{\infty} H_{b_{i}}\left(W\left(e_{i}\right)\right)\right] \\
& =a ! \cdot \prod_{i=1}^{\infty} E\left[H_{a_{i}}\left(W\left(e_{i}\right)\right) H_{b_{i}}\left(W\left(e_{i}\right)\right)\right]= \begin{cases}1 & \text { if } a=b, \\
0 & \text { otherwise } .\end{cases}
\end{aligned}
$$

Step 2: We show that $\left\{\Phi_{a}: a \in \Lambda\right\}$ forms a complete system. Assume $E\left[X \Phi_{n}\right]=0$ for all $a \in \Lambda$ for some $X \in L^{2}(\Omega, \mathcal{F} P)$ where $X \neq 0$. This means that $X$ is orthogonal to every polynomial in the variables $W\left(e_{1}\right), \ldots, W\left(e_{n}\right)$ for all $n \in \mathbb{N}$. We get

$$
E\left[X e^{i \sum_{j=1}^{n} \lambda_{j} W\left(e_{j}\right)}\right]=0, \quad \forall \lambda_{1}, \ldots, \lambda_{n} \in \mathbb{R}, n \in \mathbb{N} .
$$

The left hand side is the Fourier transform of $E\left[X \mid W\left(e_{1}\right), \ldots, W\left(e_{n}\right)\right]$ and consequently $E\left[X \mid W\left(e_{1}\right), \ldots, W\left(e_{n}\right)\right]=0$ for all $n \in \mathbb{N}$. Since $\mathcal{F}$ is generated by $W(h), h \in \mathbb{H}$ and $e_{1}, e_{2}, \ldots$ is an ON-basis in $\mathbb{H}$ it follows that $X=0$, thus $\left\{\Phi_{a}: a \in \Lambda\right\}$ must be a complete system. 
We let $\mathcal{H}_{n}$ denote the closed subspace of $L^{2}(\Omega, \mathcal{F}, P)$ spanned by $\left\{\Phi_{a}: a \in \Lambda,|a|=\right.$ $n\}$. It follows that $L^{2}(\Omega, \mathcal{F}, P)=\bigoplus_{n=0}^{\infty} \mathcal{H}_{n}$. $\mathcal{H}_{n}$ is called the $n$th Wiener Chaos. We denote by $J_{n}$ the projection operator onto the $n$th Wiener Chaos, i.e.

$$
F=\sum_{n=0}^{\infty} J_{n} F
$$

Remark 2.3.3. The $n$th Wiener chaos contains polynomials of Gaussian variables of degree $n$.

We arrived at the $n$th Wiener chaos as an expression of our ON-basis $\left(e_{i}\right)_{i=1}^{\infty}$. However the following proposition shows that the $n$th Wiener chaos is independent of the choice of this ON-basis.

Proposition 2.3.4. For each $n \geq 0$ the set of random variables

$$
\left\{H_{n}(W(h)): h \in \mathbb{H},\|h\|_{\mathbb{H}}=1\right\}
$$

is dense in $\mathcal{H}_{n}$.

Proof: The linear span of $\left\{H_{n}(W(h)): h \in \mathbb{H}, n \geq 0\right\}$ is dense in $L^{2}(\Omega, \mathcal{F}, P)$ since if a random variable $X$ is orthogonal to this set, it is orthogonal to the span of the sets $\left\{W(h)^{n}: h \in \mathbb{H}, n \geq 0\right\}$. Following the procedure of Proposition 2.3.2 we see that in this case $X=0$. Letting $\mathcal{H}_{n}^{\prime}$ denote the closed span of the family $\left\{H_{n}(W(h)):, h \in \mathbb{H}\right\}$ we get $L^{2}(\Omega, \mathcal{F}, P)=\bigoplus_{n=0}^{\infty} \mathcal{H}_{n}^{\prime}$. We have that $\bigoplus_{n=0}^{N} \mathcal{H}_{n}^{\prime}$ coincides with the set of polynomials of the family $\{W(h): h \in \mathbb{H}\}$ with degree less than or equal to $N$. We also have that $\bigoplus_{n=0}^{\mathbb{N}} \mathcal{H}_{n}$ is the span of the set of polynomials $\left\{W\left(e_{1}\right), W\left(e_{2}\right), \ldots\right\}$ of degree less than or equal to $N$. Since $\left(e_{i}\right)_{i=1}^{\infty}$ is an ON-basis it follows that $\bigoplus_{n=0}^{\infty} \mathcal{H}_{n}=$ $\bigoplus_{n=0}^{\infty} \mathcal{H}_{n}^{\prime}$ and the claim follows.

\subsection{Multiple stochastic integrals}

We will see that there is another representation for the $n$th Wiener chaos in the form of iterated Itô integrals. We begin with introducing a type of elementary functions first described by Norbert Wiener.

Definition 2.4.1. We define $\mathcal{E}_{n}$ as the set of all elementary functions of form

$$
f\left(t_{1}, \ldots, t_{n}\right)=\sum_{i_{1}, \ldots, i_{n}=1}^{k} a_{i_{1} \ldots i_{n}} \mathbb{1}_{A_{i_{1}} \times \cdots \times A_{i_{n}}}\left(t_{1}, \ldots, t_{n}\right),
$$

where $a_{i_{1} \ldots i_{n}} \in \mathbb{R}$ and $a_{i_{1} \ldots i_{n}}=0$ if two indices coincide and $A_{1}, \ldots, A_{k}$ are pairwise disjoint subsets of $[0,1]$ with finite Lebesgue measure.

We see that this is the usual simple functions in $[0,1]^{n}$ with the condition of being 0 on any diagonal described by two equal coordinates. It can be shown that this set has measure 0 and we get the following result. 
Proposition 2.4.2. The set of elementary functions $\mathcal{E}_{n}$ is dense in $L^{2}\left([0,1]^{n}\right)$.

Proof: We show that any characteristic function $\mathbb{1}_{A}$ in $L^{2}\left([0,1]^{n}\right)$ where $A=A_{1} \times \cdots \times$ $A_{n}, A_{1}, \ldots, A_{n} \in \mathcal{B}([0,1])$ can be approximated by a function in $\mathcal{E}_{n}$. Given $\varepsilon>0$ we can find $B_{1}, \ldots, B_{k} \in \mathcal{B}([0,1])$ such that $\left|B_{i}\right|<\varepsilon, i=1, \ldots, k$, the sets $B_{i}$ are disjoint, and every $A_{i}$ can be written as a union of a collection of the sets $B_{i}$. We have

$$
\mathbb{1}_{A}=\sum_{i_{1}, \ldots, i_{n}=1}^{k} a_{i_{1}, \ldots, i_{n}} \mathbb{1}_{B_{i_{1}} \times \cdots \times B_{i_{n}}}
$$

where $a_{i_{1}, \ldots, i_{n}}$ is either 0 or 1 for each multiindex $i$. We divide this sum into two parts. We let $I$ be the set of $n$-tuples $\left(i_{1}, \ldots, i_{n}\right)$ where all the indices are different and $J$ be the remaining set of $n$-tuples. We define

$$
\mathbb{1}_{B}=\sum_{\left(i_{1}, \ldots, i_{n}\right) \in I}^{k} a_{i_{1}, \ldots, i_{n}} \mathbb{1}_{B_{i_{1}} \times \cdots \times B_{i_{n}}} .
$$

Now $I_{B}$ belongs to $\mathcal{E}_{n}$ and we have

$$
\begin{aligned}
\left\|\mathbb{1}_{A}-\mathbb{1}_{B}\right\|_{L^{2}\left([0,1]^{n}\right)} & \left\|\sum_{\left(i_{1}, \ldots, i_{n}\right) \in J} a_{i_{1} \ldots i_{n}} \mathbb{1}_{B_{i_{1}} \times \cdots \times B_{i_{n}}}\right\|_{L^{2}\left([0,1]^{n}\right)} \\
& =\sum_{\left(i_{1}, \ldots, i_{n}\right) \in J} a_{i_{1} \ldots i_{n}} \mu\left(B_{i_{1}}\right) \cdot \ldots \cdot \mu\left(B_{i_{n}}\right) \\
& \leq\left(\begin{array}{l}
n \\
2
\end{array}\right) \sum_{i=1}^{k}\left(\mu\left(B_{i}\right)^{2}\right)\left(\sum_{i=1}^{k} \mu\left(B_{i}\right)\right)^{k-2}
\end{aligned}
$$

where the last inequality follows from the facts that $\left(\begin{array}{c}n \\ 2\end{array}\right)$ is loosely the number of diagonals, $\mu\left(B_{i}\right)$ is the thickness of it and $\left(\sum_{i=1}^{k} \mu\left(B_{i}\right)\right)^{k-2}$ is an upper bound of its length. Since $B_{i}$ is in $\mathcal{B}[0,1], i=1, \ldots, k$ and $\mu\left(B_{i}\right)<\varepsilon$ we get

$$
\left(\begin{array}{l}
n \\
2
\end{array}\right) \sum_{i=1}^{k}\left(\mu\left(B_{i}\right)^{2}\right)\left(\sum_{i=1}^{k} \mu\left(B_{i}\right)\right)^{k-2} \leq\left(\begin{array}{l}
n \\
2
\end{array}\right) \varepsilon\left(\sum_{i=1}^{k} \mu\left(B_{i}\right)\right)^{k-1} \leq\left(\begin{array}{l}
n \\
2
\end{array}\right) \varepsilon .
$$

Now since we can approximate characteristic functions it follows that simple functions can be approximated. As simple functions are dense in $L^{2}$ the statement follows.

We can now define a stochastic integral for any elementary function. For a function of the form (2.3) we define

$$
I_{n}\left(f\left(t_{1}, \ldots, t_{n}\right)\right)=\sum_{i_{1}, \ldots, i_{n}=1}^{k} a_{i_{1}, \ldots, i_{n}} W\left(A_{i_{1}}\right) \cdot \ldots \cdot W\left(A_{i_{n}}\right) .
$$

Remark 2.4.3. $I_{n}(f)$ can be expressed as an iterated Itô integral

$$
I_{n}(f)=n ! \int_{t_{n}=0}^{1} \int_{t_{n-1}=0}^{t_{n}} \ldots \int_{t_{1}=0}^{t_{2}} f\left(t_{1}, \ldots, t_{n}\right) d W_{t_{1}} \ldots d W_{t_{n}} .
$$


The following proposition shows some of the properties of this stochastic integral.

Proposition 2.4.4. $I_{n}$ has the following properties:

(i) $I_{n}(f)=I_{n}(\tilde{f})$, where $\tilde{f}$ denotes the symmetrization of $f$, i.e.

$$
\tilde{f}\left(t_{1}, \ldots, t_{n}\right)=\frac{1}{n !} \sum_{\Pi} f\left(t_{\Pi(1)}, \ldots, t_{\Pi(n)}\right)
$$

where $\Pi$ runs over all permutations of $\{1, \ldots, n\}$.

(ii)

$$
E\left[I_{m}(f), I_{n}(g)\right]= \begin{cases}0 & \text { if } m \neq n \\ n !\langle\tilde{f}, \tilde{g}\rangle_{L^{2}\left([0,1]^{n}\right)} & \text { if } m=n\end{cases}
$$

Proof: (i) Assume that $f$ has the form in (2.3), i.e.

$$
f\left(t_{1}, \ldots, t_{n}\right)=\sum_{i_{1}, \ldots, i_{n}=1}^{k} a_{i_{1} \ldots i_{n}} \mathbb{1}_{A_{i_{1}} \times \cdots \times A_{i_{n}}}\left(t_{1}, \ldots, t_{n}\right)
$$

The property follows immediately since

$$
\begin{aligned}
f\left(t_{\Pi(1)}, \ldots, t_{\Pi(n)}\right) & =\sum_{i_{1}, \ldots, i_{n}=1}^{k} a_{\Pi\left(i_{1}\right) \ldots \Pi\left(i_{n}\right)} \mathbb{1}_{A_{\Pi\left(i_{1}\right)} \times \cdots \times A_{\Pi\left(i_{n}\right)}}\left(t_{1}, \ldots, t_{n}\right) \\
& =\sum_{i_{1}, \ldots, i_{n}=1}^{k} a_{i_{1} \ldots i_{n}} \mathbb{1}_{A_{i_{1}} \times \cdots \times A_{i_{n}}}\left(t_{1}, \ldots, t_{n}\right) .
\end{aligned}
$$

(ii) We may assume that $f$ and $g$ are both associated with the same partition $A_{1}, \ldots, A_{k}$ otherwise we may refine the partition to satisfy the assumption. Let

$$
g\left(t_{1}, \ldots, t_{n}\right):=\sum_{i_{1}, \ldots, i_{n}=1}^{k} b_{i_{1} \ldots i_{n}} \mathbb{1}_{A_{i_{1}} \times \cdots \times A_{i_{n}}}\left(t_{1}, \ldots, t_{n}\right)
$$

Assume $m \neq n$. Using (i) we get

$$
\begin{aligned}
E\left[I_{m}(f) I_{n}(g)\right] & =E\left[I_{m}(\tilde{f}) I_{n}(\tilde{g})\right] \\
& =E\left[\sum_{\substack{k \\
i_{1}, \ldots, i_{m}=1, j_{1}, \ldots, j_{n}=1}}^{k} \tilde{a}_{i_{1} \ldots i_{m}} \tilde{b}_{j_{1} \ldots j_{n}} W\left(A_{i_{1}}\right) \cdot \ldots \cdot W\left(A_{j_{n}}\right)\right]=0
\end{aligned}
$$


since at least two of the sets $A_{i_{1}} \ldots A_{j_{n}}$ are disjoint. Now for $m=n$ we get

$$
\begin{aligned}
E\left[I_{n}(f) I_{n}(g)\right] & =E\left[I_{m}(\tilde{f}) I_{n}(\tilde{g})\right] \\
& =E\left[\sum_{i_{1}<i_{2}<\cdots<i_{n}}(n !)^{2} \tilde{a}_{i_{1} \ldots i_{n}} \tilde{b}_{i_{1} \ldots i_{n}} W\left(A_{i_{1}}\right) \cdot \ldots \cdot W\left(A_{i_{n}}\right)\right] \\
& =(n !)^{2} \sum_{i_{1}<i_{2}<\cdots<i_{n}} \tilde{a}_{i_{1} \ldots i_{n}} \tilde{b}_{i_{1} \ldots i_{n}} \mu\left(A_{i_{1}}\right) \cdot \ldots \cdot \mu\left(A_{i_{n}}\right) \\
& =n ! \sum_{i_{1}, \ldots, i_{n}=1}^{k} \tilde{a}_{i_{1} \ldots i_{n}} \tilde{b}_{i_{1} \ldots i_{n}} \mu\left(A_{i_{1}}\right) \cdot \ldots \cdot \mu\left(A_{i_{n}}\right) \\
& =n !\langle\tilde{f}, \tilde{g}\rangle_{L^{2}\left([0,1]^{n}\right) .}
\end{aligned}
$$

It follows that we get an isometry between $L_{s}^{2}\left([0,1]^{n}\right):=\left\{\tilde{f}: f \in L^{2}\left([0,1]^{n}\right)\right\}$ and $\mathcal{H}_{n}$, i.e.

$$
\sqrt{n !}\|\tilde{f}\|_{L^{2}\left([0,1]^{n}\right)}=\|I(f)\|_{L^{2}(\Omega, \mathcal{F}, P)} .
$$

Since $\mathcal{E}_{n}$ is dense in $L^{2}\left([0,1]^{n}\right)$ we can extend $I_{n}$ to a linear continuous operator from $L^{2}\left([0,1]^{n}\right)$ to $L^{2}(\Omega, \mathcal{F}, P)$.

Remark 2.4.5. The image of $L_{s}^{2}\left([0,1]^{n}\right)$ under $I_{n}$ is the $n$th Wiener chaos $\mathcal{H}_{n}$ since $I_{n}(f)$ is a polynomial of degree $n$ in $W\left(A_{1}\right), \ldots, W\left(A_{n}\right)$ when $f$ has the form (2.3), and Proposition 2.4.4(ii) shows that stochastic integrals of different order are orthogonal. The claim then follows from induction.

Since we can express a random variable in its chaos expansion we note the following:

Remark 2.4.6. If $F \in L^{2}(\Omega, \mathcal{F}, P)$, then there exists $f_{n} \in L_{s}^{2}\left([0,1]^{n}\right), n \in \mathbb{N}$ such that

$$
F=E[F]+\sum_{n=1}^{\infty} I_{n}\left(f_{n}\right) .
$$

\subsection{Derivative operator}

To define the calculus on the Wiener space a natural way of proceeding is to first look at a simple class of functions. Let $Z$ denote the set of smooth cylindrical functions

$$
Z=\left\{F: F=f\left(W\left(h_{1}\right), \ldots, W\left(h_{n}\right)\right), h_{1}, \ldots, h_{n} \in \mathbb{H}, f \in C_{p}^{\infty}\right\}
$$

where $C_{p}^{\infty}$ denotes smooth functions with polynomial growth.

Definition 2.5.1. The gradient of a smooth cylindrical function is defined by:

$$
D F:=\sum_{n=1}^{n} \frac{\partial f}{\partial x_{i}}\left(W\left(h_{1}\right), \ldots, W\left(h_{n}\right)\right) \cdot h_{i} .
$$


Remark 2.5.2. We have that $D W(h)=h$.

Clearly we have the product rule $D(F G)=D F \cdot G+F \cdot D G$ and we also get an integration by parts formula as will be shown shortly. We also define the directional derivative in the following manner.

Definition 2.5.3. The directional derivative $D_{h}$ is defined by

$$
D_{h} F:=\langle D F, h\rangle \quad h \in \mathbb{H} .
$$

This definition of the gradient $D$ gives the following integration by parts formula

Proposition 2.5.4. Suppose $F \in Z$ and $h \in \mathbb{H}$. Then $E\left[\langle D F, h\rangle_{\mathbb{H}}\right]=E[F \cdot W(h)]$.

Proof: First we assume $h=e_{1}$ and $F=f\left(W\left(e_{1}\right), \ldots, W\left(e_{n}\right)\right)$ where $f \in C_{p}^{\infty}$. Now let $\Phi$ denote the density of a standard $n$-dimensional normal distribution, i.e.

$$
\Phi\left(x_{1}, \ldots, x_{n}\right)=(2 \pi)^{-n / 2} \exp \left\{-\frac{1}{2} \sum_{i=1}^{n} x_{i}^{2}\right\} .
$$

We have

$$
\begin{aligned}
E\left[\langle D F, h\rangle_{\mathbb{H}}\right] & =E\left[\frac{\partial f}{\partial x_{1}}\left(W\left(e_{1}\right), \ldots, W\left(e_{n}\right)\right)\right]=\int_{\mathbb{R}^{n}} \frac{\partial f}{\partial x_{1}} \Phi d x \\
& \left.=\int_{\mathbb{R}^{n}} f \Phi x_{1} d x=E\left[F \cdot W\left(e_{1}\right)\right]\right)=E[F \cdot W(h)] .
\end{aligned}
$$

A change of basis proves it in the general case.

We would like to extend $D$ to an operator from $L^{2}(\Omega, \mathcal{F}, P)$ to $L^{2}(\Omega, \mathcal{F}, P ; \mathbb{H})$ and do so by first stating the next lemma.

Lemma 2.5.5. Let $F, G \in Z$ and $h \in \mathbb{H}$. Then

$$
E\left[G\langle D F, h\rangle_{\mathbb{H}}\right]=E\left[-F\langle D G, h\rangle_{\mathbb{H}}+F G W(h)\right] .
$$

Proof: Using the product rule and Proposition 2.5.4 we get:

$$
\begin{aligned}
E\left[G\langle D F, h\rangle_{\mathbb{H}}\right] & =E\left[\langle G \cdot D F, h\rangle_{\mathbb{H}}\right]=E\left[\langle D(F G)-F \cdot D G, h\rangle_{\mathbb{H}}\right] \\
& =E\left[-F\langle D G, h\rangle_{\mathbb{H}}\right]+E\left[\langle D(F G), h\rangle_{\mathbb{H}}\right] \\
& =E\left[-F\langle D G, h\rangle_{\mathbb{H}}\right]+E[F G \cdot W(h)] .
\end{aligned}
$$

Using this lemma we prove the following proposition.

Proposition 2.5.6. The operator $D$ is closable from $L^{p}(\Omega, \mathcal{F}, P)$ to $L^{p}(\Omega, \mathcal{F}, P ; \mathbb{H})$ for any $p \geq 1$.

Proof: Let $F_{n} \in Z, n=1,2, \ldots$ such that: 
- $F_{n} \rightarrow 0$ in $L^{p}$,

- $D F_{n}$ is Cauchy in $L^{p}(\Omega, \mathcal{F}, P ; \mathbb{H})$.

Let $\eta:=\lim _{n \rightarrow \infty} D F_{n}$. Left to prove is $\eta=0$. Let $h \in \mathbb{H}$ and $G=g\left(W\left(h_{1}\right), \ldots, W\left(h_{n}\right)\right)$, $g \in C_{b}^{\infty}\left(\mathbb{R}^{n}\right)$ be any smooth random variable s.t. $G W(h)$ is bounded. These $G$ are dense in $L^{p}(\Omega, \mathcal{F}, P ; \mathbb{H})$. It follows using the above lemma

$$
\begin{aligned}
E\left[\langle\eta, h\rangle_{\mathbb{H}} G\right] & =\lim _{n \rightarrow \infty} E\left[\left\langle D F_{n}, h\right\rangle_{\mathbb{H}} G\right] \\
& =\lim _{n \rightarrow \infty} E\left[-F_{n}\langle D G, h\rangle_{\mathbb{H}}+F_{n} G W(h)\right]=0 .
\end{aligned}
$$

It follows $\langle\eta, h\rangle_{\mathbb{H}}=0$ and since $h$ was arbitrary we get $\eta=0$.

As a consequence there exists a unique closed extension $\left(D, \mathbb{D}^{1, p}\right)$ of $(D, Z)$. Here the case $p=2$ is mostly considered. The closability of $D$ gives rise to the chain rule

Proposition 2.5.7. (Chain rule) Let $\varphi: \mathbb{R}^{m} \rightarrow \mathbb{R}$ be a continuously differentiable function with bounded partial derivatives. Suppose $F=\left(F_{1}, \ldots, F_{m}\right) \in\left(\mathbb{D}^{1, p}\right)^{m}$ for some $p \geq 1$. Then $\varphi(F) \in \mathbb{D}^{1, p}$ and

$$
D(\varphi(F))=\sum_{i=1}^{m} \frac{\partial \varphi}{\partial x_{i}}(F) D F_{i} .
$$

Proof: Since $\mathbb{D}^{1, p}$ is closed we can find sequences $F_{i}^{(n)} \in Z, i=1, \ldots, m$ such that $F_{i}^{(n)} \underset{n \rightarrow \infty}{\longrightarrow} F_{i}$, in $L^{p}$, and $D F_{i}^{(n)} \underset{n \rightarrow \infty}{\longrightarrow} D F_{i}$, in $L^{p}(\Omega, \mathcal{F}, P ; H)$. The claim follows.

Taking the gradient of a function lowers the corresponding degree with respect to the Wiener chaos in a similar fashion as taking the derivative of a polynomial lowers the degree by one. The following two propositions describe this relation in detail.

Proposition 2.5.8. Let $F \in L^{2}(\Omega, \mathcal{F}, P)$ with $F=\sum_{i=0}^{\infty} J_{n} F$ corresponding to the Wiener chaos decomposition. Then $F \in \mathbb{D}^{1,2}$ if and only if

$$
E\left[\|D F\|_{\mathbb{H}}^{2}\right]=\sum_{n=1}^{\infty} n\left\|J_{n} F\right\|_{2}^{2}<\infty .
$$

In this case, for all $n \in \mathbb{N}$ and $h \in \mathbb{H}$ we have

$$
\left\langle D\left(J_{n} F\right), h\right\rangle_{\mathbb{H}}=J_{n-1}\left(\langle D F, h\rangle_{\mathbb{H}}\right) .
$$

Proposition 2.5.9. Let $F \in \mathbb{D}^{1,2}$ and assume that $F$ has representation

$$
F=E[F]+\sum_{n=1}^{\infty} I_{n}\left(f_{n}\right)
$$

where $f_{n} \in L_{s}^{2}\left([0,1]^{n}\right)$. Then

$$
D_{t} F=\sum_{n=1}^{\infty} n I_{n-1}\left(f_{n}(\cdot, t)\right), \quad t \in[0,1] .
$$


Proof: First let $F=I_{n}\left(f_{n}\right)$ where

$$
f_{n}\left(t_{1}, \ldots, t_{n}\right)=\sum_{i_{1}, \ldots, i_{n}}^{k} a_{i_{1} \ldots i_{n}} \mathbb{1}_{A_{i_{1}} \times \cdots \times A_{i_{n}}}\left(t_{1}, \ldots, t_{n}\right)
$$

Then

$$
F=I_{n}\left(f_{n}\right)=\sum_{i_{1}, \ldots, i_{n}=1}^{k} a_{i_{1} \ldots i_{n}} W\left(A_{i_{1}}\right) \cdot \ldots \cdot W\left(A_{i_{n}}\right)
$$

and $F \in Z$. We get

$$
D_{t} F=\sum_{j=1}^{n} \sum_{i_{1}, \ldots, i_{n}=1}^{k} a_{i_{1} \ldots i_{n}} W\left(A_{i_{1}}\right) \cdot \ldots \cdot \mathbb{1}_{A_{i_{j}}}(t) \cdot \ldots \cdot W\left(A_{i_{n}}\right)=n I_{n-1}(f(\cdot, t))
$$

by symmetry. The claim follows from the closedness of $\mathbb{D}^{1,2}$.

Suppose that $F$ is given. How does one find the representation of $F$ ? The following proposition provides a tool to achieve this.

Proposition 2.5.10. If $F \in \mathbb{D}^{\infty, 2}$ and

$$
F=E[F]+\sum_{n=1}^{\infty} I_{n}\left(f_{n}\right)
$$

then

$$
f_{k}=\frac{1}{k !} E\left[D^{k} F\right], \quad K \in \mathbb{N} \text {. }
$$

Proof: Applying Proposition 2.5.9 $k$ times yields

$$
D_{t_{1}, \ldots, t_{k}}^{k} F=\sum_{n=k}^{\infty} n(n-1) \ldots(n-k+1) I_{n-k}\left(f_{n}\left(t_{1}, \ldots, t_{k} ; \cdot\right)\right)
$$

and thus

$$
\frac{1}{k !} D_{t_{1}, \ldots, t_{k}}^{k} F=I_{0}\left(f_{k}\left(t_{1}, \ldots, t_{k}\right)\right)+\sum_{n=1}^{\infty} I_{n}\left(g_{n}\right)=f_{k}\left(t_{1}, \ldots, t_{k}\right)+\sum_{n=1}^{\infty} I_{n}\left(g_{n}\right)
$$

where $g_{n}$ is the representing function of the chaos expansion of $\frac{1}{k !} D_{t_{1}, \ldots, t_{k}}^{k} F$. It follows that

$$
\frac{1}{k !} E\left[D_{t_{1}, \ldots, t_{k}}^{k} F\right]=f_{k}\left(t_{1}, \ldots, t_{k}\right) \text {. }
$$

The following example illustrates the usage of the above proposition. 


\section{Example 2.2}

Let $W_{t}, t \in[0,1]$ be a one dimensional Wiener process. The Wiener chaos expansion of $F=W_{a}^{2} W_{1}, 0 \leq a \leq 1$ is then

$$
\begin{aligned}
W_{a}^{2} W_{1} & =2 a W_{a}+a W_{1}+4 \int_{t_{3}=0}^{a} \int_{t_{2}=0}^{t_{3}} \int_{t_{1}=0}^{t_{2}} d W_{t_{1}} d W_{t_{2}} d W_{t_{3}} \\
& +2 \int_{t_{3}=0}^{1} \int_{t_{2}=0}^{t_{3}} \int_{t_{1}=0}^{t_{2}} \mathbb{1}_{[0, a]}\left(t_{1} \vee t_{2}\right) d W_{t_{1}} d W_{t_{2}} d W_{t_{3}} .
\end{aligned}
$$

We note that $W_{a}^{2} W_{1}=W\left(\mathbb{1}_{[0, a]}\right)^{2} W\left(\mathbb{1}_{[0,1]}\right)$ and use Proposition 2.5.10 to obtain

$$
\begin{gathered}
f_{0}=E\left[W_{a}^{2} W_{1}\right]=0, \\
f_{1}\left(t_{1}\right)=E\left[D_{t_{1}} W_{a}^{2} W_{1}\right]=E\left[D W_{a}^{2} W_{1}\right]_{t_{1}} \\
=E\left[2 W\left(\mathbb{1}_{[0, a]}\right) W\left(\mathbb{1}_{[0,1]}\right) \mathbb{1}_{[0, a]}\left(t_{1}\right)+W\left(\mathbb{1}_{[0, a]}\right)^{2} \mathbb{1}_{[0,1]}\left(t_{1}\right)\right] \\
=2 E\left[W_{a} W_{1} \mathbb{1}_{[0, a]}\left(t_{1}\right)+W_{a}^{2} \mathbb{1}_{[0,1]}\left(t_{1}\right)\right]=2 a \mathbb{1}_{[0, a]}\left(t_{1}\right)+a, \\
f_{2}\left(t_{1}, t_{2}\right)=\frac{1}{2} E\left[D_{t_{1}, t_{2}}^{2} W_{a}^{2} W_{1}\right]=\ldots \\
=E\left[W_{1}\right] \mathbb{1}_{[0, a]}\left(t_{1} \vee t_{2}\right)+E\left[W_{a}\right]\left(\mathbb{1}_{[0,1]}\left(t_{1}\right)+\mathbb{1}_{[0, a]}\left(t_{2}\right)\right)=0,
\end{gathered}
$$

where the ... signifies using the elementary operations as for calculation of $f_{1}$. In the same manner we get

$$
\begin{aligned}
f_{3}\left(t_{1}, t_{2}, t_{3}\right) & =\frac{1}{3 !} E\left[D_{t_{1}}\left(D_{t_{2}}\left(D_{t_{3}} W_{a}^{2} W_{1}\right)\right)\right]=\ldots \\
& =\frac{1}{3}\left(\mathbb{1}_{[0, a]}\left(t_{2} \vee t_{3}\right)+\mathbb{1}_{[0, a]}\left(t_{1} \vee t_{2}\right)+\mathbb{1}_{[0, a]}\left(t_{1} \vee t_{3}\right)\right) .
\end{aligned}
$$

We recall

$$
F=\int_{0}^{1} f_{1}\left(t_{1}\right) d W_{t_{1}}+3 ! \int_{t_{3}=0}^{1} \int_{t_{2}=0}^{t_{3}} \int_{t_{1}=0}^{t_{2}} f_{3}\left(t_{1}, t_{2}, t_{3}\right) d W_{t_{1}} d W_{t_{2}} d W_{t_{3}}
$$

and the statement follows.

\subsection{Divergence operator}

We let Dom $\delta$ be the set of all $u \in L^{2}(\Omega, \mathcal{F}, P ; \mathbb{H})$ such that there exists $c(u)>0$ with

$$
\left|E\left[\langle D F, u\rangle_{\mathbb{H}}\right]\right| \leq c\|F\|_{L^{2}}
$$


for all $F \in \mathbb{D}^{1,2}$. Then $E\left[\langle D F, u\rangle_{\mathbb{H}}\right]$ is a bounded linear functional from $\mathbb{D}^{1,2}$ to $\mathbb{R}$. Now since $\mathbb{D}^{1,2}$ is dense in $L^{2}(\Omega, \mathcal{F}, P)$ Riesz representation theorem states that there exists a unique representing element $\delta(u)$, bounded in $L^{2}(\Omega, \mathcal{F}, P)$. That is

$$
E\left[\langle D F, u\rangle_{\mathbb{H}}\right]=E[F \delta(u)], \quad \forall F \in \mathbb{D}^{1,2}
$$

In other words $\delta: L^{2}(\Omega, \mathcal{F}, P ; \mathbb{H}) \rightarrow L^{2}$ and $\delta$ is the adjoint operator to $D$. We call $\delta$ the divergence operator.

Proposition 2.6.1. The divergence operator $(\delta, \operatorname{Dom} \delta)$ has the following properties (i)

$$
E[\delta(u)]=0 \quad \forall u \in \operatorname{Dom} \delta .
$$

(ii) Suppose $u \in Z_{\mathbb{H}}:=\left\{v=\sum_{j=1}^{n} F_{j} h_{j}: F_{j} \in Z, h_{j} \in \mathbb{H}\right\}$, then $u \in \operatorname{Dom} \delta$ and

$$
\delta(u)=\sum_{j=1}^{n} F_{j} W\left(h_{j}\right)-\sum_{j=1}^{n}\left\langle D F_{j}, h_{j}\right\rangle_{\mathbb{H}} \cdot
$$

(iii) The operator $(\delta, \operatorname{Dom} \delta)$ is closed, i.e. if $u_{n} \in \operatorname{Dom} \delta, n=1,2, \ldots, u_{n} \rightarrow u$ in $L^{2}(\Omega, \mathcal{F}, P ; \mathbb{H})$, and $\delta\left(u_{n}\right) \rightarrow G$ in $L^{2}$, then $u \in \operatorname{Dom} \delta$ and $\delta(u)=G$.

Proof: (i) This follows from putting $F \equiv 1$ in (2.5).

(ii) Using Lemma 2.5.5 we have

$$
\begin{aligned}
\left|E\left[\langle D F, u\rangle_{\mathbb{H}}\right]\right| & =\left|E\left[\sum_{j=1}^{n} F_{j}\left\langle D F, h_{j}\right\rangle_{\mathbb{H}}\right]\right| \\
& =\left|E\left[F\left(\sum_{j=1}^{n}-\left\langle D F_{j}, h_{j}\right\rangle_{\mathbb{H}}+\sum_{j=1}^{n} F_{j} W\left(h_{j}\right)\right)\right]\right| \leq c(u)\|F\|_{L^{2}}
\end{aligned}
$$

so $u \in \operatorname{Dom} \delta$. We also get

$$
E\left[\langle D F, u\rangle_{\mathbb{H}}\right]=E\left[F\left(\sum_{j=1}^{n}-\left\langle D F_{j}, h_{j}\right\rangle_{\mathbb{H}}+\sum_{j=1}^{n} F_{j} W\left(h_{j}\right)\right)\right]=E[F \delta(u)]
$$

and the statement follows.

(iii) Let $u_{n} \rightarrow u$ in $\left.L^{2}(\Omega, \mathcal{F}, P)\right)$. Then for any $F \in \mathbb{D}^{1,2}$ we have

$$
E\left[F \delta\left(u_{n}\right)\right]=E\left[\left\langle D F, u_{n}\right\rangle_{\mathbb{H}}\right] \underset{n \rightarrow \infty}{\longrightarrow} E\left[\langle D F, u\rangle_{\mathbb{H}}\right]=E[F \delta(u)]
$$

Since $F$ is arbitrary it follows that $G=\delta(u)$, and also $u \in \operatorname{Dom} \delta$ since (2.4) is satisfied with $c(u)=\|G\|_{L^{2}}$.

The following formulas state the relation between divergence $\delta$ and gradient $D$. 
Proposition 2.6.2. Let $u, v \in Z_{\mathbb{H}}, F \in Z$ and $h \in \mathbb{H}$. Let $\left\{e_{i}: i \in \mathbb{N}\right\}$ be an ON-basis in $\mathbb{H}$. Then

a) $D_{h}(\delta(u))=\langle u, h\rangle_{\mathbb{H}}+\delta\left(D_{h}(u)\right)$.

b) $E[\delta(u) \delta(v)]=E\left[\langle u, v\rangle_{\mathbb{H}}\right]+E\left[\sum_{i, j=1}^{\infty} D_{e_{i}}\left\langle u, e_{j}\right\rangle_{\mathbb{H}} D_{e_{j}}\left\langle v, e_{i}\right\rangle_{\mathbb{H}}\right]$.

c) $\delta(F u)=F \delta(u)-\langle D F, u\rangle_{\mathbb{H}}$.

Proof: a) Let $u=\sum_{j=1}^{n} F_{j} h_{j}$ where $F_{j} \mathbb{Z}, h_{j} \in \mathbb{H}, j=1, \ldots, n$. Now

$$
\begin{aligned}
D_{h}(\delta(u)) & =\langle D(\delta(u)), h\rangle_{\mathbb{H}}=\langle D \overbrace{\left(\sum_{j=1}^{n} F_{j} W\left(h_{j}\right)-\sum_{j=1}^{n}\left\langle D F_{j}, h_{j}\right\rangle_{\mathbb{H}}\right)}^{\text {Proposition 2.6.1(ii) }}, h\rangle_{\mathbb{H}} \\
& =\left\langle\sum_{j=1}^{n} F_{j} D W\left(h_{j}\right)+\sum_{j=1}^{n} D F_{j} W\left(h_{j}\right)-\sum_{j=1}^{n} D\left(\left\langle D F_{j}, h_{j}\right\rangle_{\mathbb{H}}\right), h\right\rangle_{\mathbb{H}} \\
& =\sum_{j=1}^{n} F_{j}\left\langle h, h_{j}\right\rangle_{\mathbb{H}}+\sum_{j=1}^{n} D_{h} F_{j} W\left(h_{j}\right)-\sum_{j=1}^{n} D_{h}\left(\left\langle D F_{j}, h_{j}\right\rangle_{\mathbb{H}}\right) \\
& \left.=\langle u, h\rangle_{\mathbb{H}}+\sum_{j=1}^{n}\left(D_{h} F_{j}\right) W\left(h_{j}\right)-\sum_{j=1}^{n}\left\langle D\left(D_{h} F_{j}\right), h_{j}\right\rangle_{\mathbb{H}}\right) \\
& =\langle u, h\rangle_{\mathbb{H}}+\delta\left(D_{h}(u)\right)
\end{aligned}
$$

where we in the last line once again used Proposition 2.6.1(ii).

b) Using a) we obtain

$$
\begin{aligned}
E[\delta(u) \delta(v)] & =E\left[\langle u, D \delta(v)\rangle_{\mathbb{H}}\right]=E\left[\sum_{i=1}^{\infty}\left\langle u, e_{i}\right\rangle_{\mathbb{H}} D_{e_{i}} \delta(v)\right] \\
& =E\left[\sum_{i=1}^{\infty}\left\langle u, e_{i}\right\rangle_{\mathbb{H}}\left(\left\langle v, e_{i}\right\rangle_{\mathbb{H}}+\delta\left(D_{e_{i}}(v)\right)\right)\right] \\
& =E\left[\langle u, v\rangle_{\mathbb{H}}\right]+\sum_{i=1}^{\infty} E\left[\left\langle u, e_{i}\right\rangle_{\mathbb{H}} \delta\left(\left\langle D v, e_{i}\right\rangle_{\mathbb{H}}\right)\right] \\
& =E\left[\langle u, v\rangle_{\mathbb{H}}\right]+E\left[\sum_{i=1}^{\infty}\left\langle\sum_{j=1}^{\infty} D_{e_{j}}\left\langle u, e_{i}\right\rangle_{\mathbb{H}} e_{j}, D_{e_{i}}\left(\sum_{k=1}^{\infty}\left\langle v, e_{k}\right\rangle e_{k}\right)\right\rangle\right] \\
& =E\left[\langle u, v\rangle_{\mathbb{H}}\right]+E\left[\sum_{i, j=1}^{\infty} D_{e_{j}}\left\langle u, e_{i}\right\rangle_{\mathbb{H}} \cdot D_{e_{i}}\left\langle v, e_{j}\right\rangle_{\mathbb{H}}\right]
\end{aligned}
$$


c) For $G \in Z$ we have

$$
\begin{aligned}
E[G \delta(F u)] & =E\left[\langle D G, F u\rangle_{\mathbb{H}}=E\left[\langle F D G, u\rangle_{\mathbb{H}}\right]=E\left[\langle u, D(F G)-G D F\rangle_{\mathbb{H}}\right]\right. \\
& =E\left[\left(\delta(u) F-\langle u, D F\rangle_{\mathbb{H}}\right) G\right] .
\end{aligned}
$$

Since $G$ was arbitrary it follows $\delta(F u)=F \delta(u)-\langle D F, u\rangle_{\mathbb{H}}$.

We state the connection between the Wiener chaos expansion and the divergence operator. Recall that for $u \in L^{2}([0,1] \times \Omega), u$ has a Wiener chaos expansion of form

$$
u(t)=\sum_{n=0}^{\infty} I_{n}\left(f_{n}(\cdot, t)\right),
$$

where $f_{n} \in L^{2}\left([0,1]^{n}\right)$ and $f_{n}$ is symmetric in the $n$ first variables.

Proposition 2.6.3. We have $u \in \operatorname{Dom} \delta$ if and only if the series

$$
\sum_{n=0}^{\infty} I_{n+1}\left(\tilde{f}_{n}\right)
$$

converges in $L^{2}(\Omega, \mathcal{F}, P)$. In this case we have

$$
\delta(u)=\sum_{n=0}^{\infty} I_{n+1}\left(\tilde{f}_{n}\right) .
$$

Proof: Suppose $G=I_{n}(g)$ where $g \in L_{s}^{2}\left([0,1]^{n}\right)$. Now using Proposition 2.5.9 we get

$$
\begin{aligned}
E\left[\langle u, D G\rangle_{\mathbb{H}}\right] & =E\left[\left\langle u, n I_{n-1}(g(\cdot, t))\right\rangle_{\mathbb{H}}\right]=E\left[\left\langle I_{n-1}\left(f_{n-1}(\cdot, t)\right), n I_{n-1}(g(\cdot, t))\right\rangle_{\mathbb{H}}\right] \\
& =\int_{[0,1]} E\left[I_{n-1}\left(f_{n-1}(\cdot, t)\right) n I_{n-1}(g(\cdot, t))\right] d t \\
& =n(n-1) ! \int_{[0,1]}\left\langle f_{n-1}, g\right\rangle_{L^{2}\left([0,1]^{n-1}\right)} d t \\
& =n !\left\langle f_{n-1}, g\right\rangle_{L^{2}\left([0,1]^{n}\right.}=n !\left\langle\tilde{f}_{n-1}, g\right\rangle_{L^{2}\left([0,1]^{n}\right)} \\
& =E\left[I_{n}\left(\tilde{f}_{n-1}\right) I_{n}(g)\right]=E\left[I_{n}\left(\tilde{f}_{n-1}\right) G\right]
\end{aligned}
$$

where the third and last lines come from the isometry between $L_{s}^{2}([0,1])^{n}$ and $\mathcal{H}_{n}$.

One can see from this that $\delta$ is an integral. $\delta(u)$ is called the Skorokhod stochastic integral of the process $u$ and if the process is adapted then the Skorokhod integral will coincide with the Itô integral, i.e.

$$
\delta(u)=\int_{0}^{1} u_{t} d W_{t} .
$$




\subsection{The generator of the Ornstein-Uhlenbeck semigroup}

The Ornstein-Uhlenbeck semigroup is related to the solution of some stochastic differential equations. It is closely related to the gradient and divergence. The generator of this semigroup is denoted $L$. In this presentation we start with the following definition.

Definition 2.7.1. For $F \in L^{2}(\Omega, \mathcal{F}, P)$ define

$$
L F:=-\sum_{n=0}^{\infty} n J_{n} F
$$

where $J_{n}$ denotes the projection to the $n$th Wiener chaos. We define Dom $L$ as

$$
\text { Dom } L:=\left\{F \in L^{2}: \sum_{n=0}^{\infty} n^{2}\left\|J_{n} F\right\|_{2}^{2}<\infty\right\} \text {. }
$$

The relation to the gradient and divergence is presented in the next propositions.

Proposition 2.7.2. For $F \in L^{2}(\Omega, \mathcal{F}, P)$ the following are equivalent:

(i) $F \in \operatorname{Dom} L$

(ii) $F \in \mathbb{D}^{1,2}$ and $D F \in \operatorname{Dom} \delta$.

If (i) or (ii) holds then $-L F=\delta D F$.

Proof: We know from earlier that for $G \in \mathbb{D}^{1,2}$ we have

$$
E\left[\langle D G, D F\rangle_{\mathbb{H}}\right]=\sum_{n=0}^{\infty} n E\left[J_{n} F J_{n} G\right]
$$

Assume that (ii) holds. Let $G \in \mathcal{H}_{n}$, and $F \in \mathbb{D}^{1,2}$, then

$$
E[G \delta(D F)]=E\left[\langle D G, D F\rangle_{\mathbb{H}}\right]=n E\left[J_{n} G J_{n} F\right]=n E\left[G J_{n} F\right] .
$$

For a general $G \in \mathbb{D}^{1,2}$ we get

$$
E[G \delta(D F)]=E\left[\left\langle D\left(\sum_{n=0}^{\infty} J_{n} G\right), D F\right\rangle_{\mathbb{H}}\right]=\sum_{n=0}^{\infty} n E\left[J_{n} G J_{n} F\right]=E\left[G \sum_{n=0}^{\infty} n J_{n} F\right] .
$$

Since $G$ was arbitrary (i) follows. Assuming (i), and letting $G \in \mathbb{D}^{1,2}$ be a test function, we get

$$
E[G \cdot L F]=-\sum_{n=0}^{\infty} E\left[G n J_{n} F\right]=-\sum_{n=0}^{\infty} n E\left[J_{n} G J_{n} F\right]=-E\left[\langle D G, D F\rangle_{\mathbb{H}}\right] .
$$

By definition $D F \in \operatorname{Dom} \delta$ and $\delta D F=-L F$. 
Proposition 2.7.3. We have $Z \in$ Dom $\delta$ and for $F \in Z$ with $F=f\left(W\left(h_{1}\right), \ldots, W\left(h_{n}\right)\right), f \in C_{p}^{\infty}\left(\mathbb{R}^{n}\right)$ we have

$$
\begin{aligned}
L F & =\sum_{i, j=1}^{n} \frac{\partial^{2}}{\partial x_{i} \partial x_{j}} f\left(W\left(h_{1}\right), \ldots, W\left(h_{n}\right)\right)\left\langle h_{i}, h_{j}\right\rangle_{\mathbb{H}} \\
& -\sum_{i}^{n} \frac{\partial}{\partial x_{i}} f\left(W\left(h_{1}\right), \ldots, W\left(h_{n}\right)\right) W\left(h_{i}\right) .
\end{aligned}
$$

Proof: Since $F \in \mathbb{D}^{1,2}$ and $D F=\sum_{i=1}^{n} \frac{\partial}{\partial x_{i}} f\left(W\left(h_{1}\right), \ldots, W\left(h_{n}\right)\right) h_{i}$ we have that $D F \in Z_{\mathbb{H}}$. We recall that for

$$
u:=\sum_{j=1}^{n} F_{j} h_{j}, \quad F_{j} \in \mathbb{D}^{1,2}, h_{j} \in \mathbb{H},
$$

we have

$$
\delta(u)=\sum_{j=1}^{n} F_{j} W\left(h_{j}\right)-\sum_{j=1}^{n}\left\langle D F_{j}, h_{j}\right\rangle_{\mathbb{H}}
$$

We now get

$$
\begin{aligned}
\delta D F= & \sum_{i=1}^{n} \frac{\partial}{\partial x_{i}} f\left(W\left(h_{1}\right), \ldots, W\left(h_{n}\right)\right) W\left(h_{i}\right) \\
& -\sum_{j=1}^{n}\left\langle D\left(\frac{\partial}{\partial x_{j}} f\left(W\left(h_{1}\right), \ldots, W\left(h_{n}\right)\right)\right), h_{j}\right\rangle_{\mathbb{H}} \\
= & \sum_{i=1}^{n} \frac{\partial}{\partial x_{i}} f \cdot W\left(h_{i}\right)-\sum_{j=1}^{n}\left\langle\sum_{i=1}^{n} \frac{\partial}{\partial x_{i}} f \cdot h_{i}, h_{j}\right\rangle_{\mathbb{H}} \\
= & \sum_{i=1}^{n} \frac{\partial}{\partial x_{i}} f \cdot W\left(h_{i}\right)-\sum_{i, j=1}^{n} \frac{\partial}{\partial x_{i} \partial x_{j}} f \cdot\left\langle h_{i}, h_{j}\right\rangle_{\mathbb{H}}
\end{aligned}
$$

and the claim follows.

We see that the Malliavin calculus provides the necessary tools for doing analysis on the Wiener space, the most prominent tool being the integration by parts formula. 


\section{Chapter 3-Dirichlet form theory}

$\mathrm{D}$ RICHLET forms are a special type of bilinear forms. The theory of Dirichlet forms was first introduced in the works by Beurling and Deny in 1958 and 1959. There is a link between Dirichlet forms and Markov processes. In this chapter the general definitions are introduced and the links between semigroup, resolvent, generator, and form are stated.

\subsection{Introduction}

This chapter follows the structure in the book by Z.-M. Ma and M. Röckner [5] and the theory can be found there in a more general form. In this section we will let $\mathbb{H}$ be a Hilbert space with corresponding norm $\|\cdot\|_{\mathbb{H}}$ and inner product $\langle\cdot, \cdot\rangle_{\mathbb{H}}$.

\subsection{Resolvents}

The resolvent set of a linear operator $L$ is a set of complex numbers $\lambda$ for which the operator $L-\lambda I$ is in some sense well-behaved.

Definition 3.2.1. Let $L$ be a linear operator on $\mathbb{H}$. The resolvent set $\rho(L)$ of $L$ is defined to be the set of all $\alpha \in \mathbb{R}$ such that $(\alpha-L): D(L) \rightarrow \mathbb{H}$ is one-to-one and for its inverse $(\alpha-L)^{-1}$ we have

(i) $D\left((\alpha-L)^{-1}\right)=\mathbb{H}$,

(ii) $(\alpha-L)^{-1}$ is continuous on $\mathbb{H}$.

We call $\left\{(\alpha-L)^{-1}: \alpha \in \rho(L)\right\}$ the resolvent of $L$ and $\sigma(L):=\mathbb{R} \backslash \rho(L)$ the spectrum of $L$.

We see that all eigenvalues are part of the spectrum but the spectrum can have other points as well. For $\alpha \in \rho(L)$ we let $G_{a}:=(\alpha-L)^{-1}$. 
Definition 3.2.2. A family $\left.\left(G_{\alpha}\right)_{\alpha>0}\right)$ of linear operators on $\mathbb{H}$ is called a continuous contraction resolvent on $\mathbb{H}$ if:

(i) $\lim _{\alpha \rightarrow \infty} \alpha G_{\alpha} u=u$ for all $u \in \mathbb{H}$,

(continuity)

(ii) $G_{a}$ is a contraction on $\mathbb{H}$ for all $\alpha>0$,

(iii) $G_{\alpha}-G_{\beta}=(\beta-\alpha) G_{\alpha} G_{\beta}$ for all $\alpha, \beta>0 . \quad$ (resolvent equation)

\subsection{Semi-groups}

In probability theory, semigroups are associated with the transition probabilities of Markov processes.

Definition 3.3.1. A family $\left(T_{t}\right)_{t>0}$ of linear operators on $\mathbb{H}$ with $D\left(T_{t}\right)=\mathbb{H}$ for all $t>0$ is called a continuous contraction semigroup on $\mathbb{H}$ if

(i) $\lim _{t \rightarrow 0} T_{t} u=u$ for all $u \in \mathbb{H}$,

(continuity)

(ii) $T_{t}$ is a contraction on $\mathbb{H}$ for all $t>0$,

(iii) $T_{s} T_{t}=T_{s+t}$ for all $s, t>0$.

(semigroup property)

It turns out that semigroups are connected to resolvents as will be shown later.

Definition 3.3.2. The infinitesimal generator of a continuous semigroup $\left(T_{t}\right)_{t>0}$ is defined as the operator $(\mathbf{A}, D(\mathbf{A}))$ on $\mathbb{H}$ satisfying

$$
\begin{aligned}
D(\mathbf{A}) & =\left\{u \in \mathbb{H}: \lim _{t \searrow 0} \frac{T_{t} u-u}{t} \text { exists }\right\}, \\
\mathbf{A} u & =\lim _{t \searrow 0} \frac{T_{t} u-u}{t}, \quad u \in D(\mathbf{A}) .
\end{aligned}
$$

Given a semigroup and thus also its generator, it is possible to, in a natural way, associate it with a resolvent. The following proposition shows this relation.

Proposition 3.3.3. Let $\left(T_{t}\right)_{t>0}$ be a continuous contraction semigroup on $\mathbb{H}$ with generator $\mathbf{A}$. Then $\mathbf{A}$ is densely defined, $(0, \infty) \subset \rho(\mathbf{A})$ and if $G_{\alpha}:=(\alpha-\mathbf{A})^{-1}, \alpha>0$ then

$$
G_{\alpha} u=\int_{0}^{\infty} e^{-\alpha s} T_{s} u d s, \quad u \in \mathbb{H}, \quad \alpha>0 .
$$

We also have that $\left(G_{\alpha}\right)_{\alpha>0}$ is a continuous contraction resolvent.

Proof: The proof of this proposition will be omitted but can be found in [5].

We see that there is a connection between semigroups, resolvents, and the generator A. For an arbitrary linear operator $L$ one might wonder if it is the generator of a semigroup or not. The answer to this quesion is answered by the Hille-Yosida theorem, which characterizes the generators for contraction semigroups. The theorem is named after the mathematicians Einar Hille and Kosaku Yoside who independently discovered the result in 1948. 
Theorem 3.3.4. (Hille-Yosida) A densely defined linear operator $\mathbf{A}$ on $\mathbb{H}$ is the generator of a continuous contraction semigroup if any only if

$$
\begin{aligned}
& \text { (i) }(0, \infty) \subset \rho(\mathbf{A}) \text {, } \\
& \text { (ii) }\left\|\alpha(\alpha-\mathbf{A})^{-1}\right\| \leq 1 \text { for all } \alpha>0 .
\end{aligned}
$$

The continuous contraction semigroup is uniquely determined by $\mathbf{A}$ and $\mathbf{A}$ is closed.

Proof: The proof of the Hille-Yosida theorem is quite lengthy and will therefore be omitted. The full proof can be found in [5].

\subsection{Bilinear forms}

In this section we'll look into the relationship between the resolvent, semigroup and bilinear form. We will assume that $\mathcal{E}$ is positive definite throughout the section.

Definition 3.4.1. The symmetric part $\tilde{\mathcal{E}}$ of a bilinear form $\mathcal{E}$ is defined by

$$
\tilde{\mathcal{E}}(u, v)=\frac{1}{2}(\mathcal{E}(u, v)+\mathcal{E}(v, u)) .
$$

Definition 3.4.2. We define

$$
\mathcal{E}_{\alpha}(u, v)=\mathcal{E}(u, v)+\alpha\langle u, v\rangle_{\mathbb{H}}, \quad u, v \in D(\mathcal{E}) .
$$

Following this definition we can also define the corresponding norm.

Definition 3.4.3. Given a bilinear form $\mathcal{E}$ on a Hilbert space $\mathbb{H}$ we define the $\mathcal{E}_{1}$-norm by

$$
\|\cdot\|_{\mathcal{E}_{1}}:=\left(\langle\cdot, \cdot\rangle_{\mathbb{H}}+\mathcal{E}(\cdot, \cdot)\right)^{1 / 2}
$$

Definition 3.4.4. $(\mathcal{E}, D(\mathcal{E}))$ satisfies the weak sector condition if there exists a constant $K>0$ such that

$$
\left|\mathcal{E}_{1}(u, v)\right| \leq K \mathcal{E}_{1}(u, u)^{1 / 2} \mathcal{E}_{1}(v, v)^{1 / 2}, \forall u, v \in D(\mathcal{E}) .
$$

Definition 3.4.5. A bilinear form $(\mathcal{E}, D(\mathcal{E}))$ on $\mathbb{H}$ is called coercive if $D(\mathcal{E})$ is a dense linear subspace of $\mathbb{H}$ and $\mathcal{E}: \mathbb{H} \times \mathbb{H} \rightarrow \mathbb{R}$ such that

(i) Its symmetric part $(\tilde{\mathcal{E}}, D(\mathcal{\varepsilon}))$ is a symmetric closed form on $\mathbb{H}$.

(ii) $(\mathcal{E}, D(\mathcal{E}))$ satisfies the weak sector condition.

A property of a coercive form is that it grows rapidly as the argument gets bigger.

Theorem 3.4.6. Let $(\mathcal{E}, D(\mathcal{E}))$ be a coercive closed form on $\mathbb{H}$. Then there exist unique continuous contraction resolvents $\left(G_{\alpha}\right)_{\alpha>0},\left(\tilde{G}_{\alpha}\right)_{\alpha>0}$ on $\mathbb{H}$ such that

$$
\begin{aligned}
& G_{\alpha}(\mathbb{H}), \tilde{G}_{\alpha}(\mathbb{H}) \subset D(\mathcal{E}), \\
& \mathcal{E}_{\alpha}\left(G_{\alpha} f, u\right)=\langle f, u\rangle_{\mathbb{H}}=\mathcal{E}_{\alpha}\left(u, \tilde{G}_{\alpha} f\right) \text { for all } f \in \mathbb{H}, u \in D(\mathcal{E}), \alpha>0 .
\end{aligned}
$$

$\tilde{G}_{\alpha}$ is the adjoint of $G_{\alpha}$ for all $\alpha>0$ and we have

$$
\left\langle G_{\alpha} f, g\right\rangle_{\mathbb{H}}=\left\langle f, \tilde{G}_{\alpha} g\right\rangle \text { for all } f, g \in \mathbb{H} \text {. }
$$


Proof: The proof of this theorem will be omitted. As in the other cases the full proof can be found in [5].

A consequence of this is the following relation between the bilinear form and the corresponding generator to the resolvent.

Corollary 3.4.7. Let $(\mathcal{E}, D(\mathcal{E}))$ and $\left(G_{\alpha}\right)_{\alpha>0}$ be as above and let $(\mathbf{A}, D(\mathbf{A}))$ be the generator of $\left(G_{\alpha}\right)_{\alpha>0}$ i.e. the unique operator on $\mathbb{H}$ such that $(0, \infty) \in \rho(\mathbf{A})$ and $G_{\alpha}=$ $(\alpha-\mathbf{A})^{-1}$ for all $\alpha>0$. Then

$$
\begin{aligned}
& D(\mathbf{A}) \subset D(\mathcal{E}), \\
& \mathcal{E}(u, v)=\langle-\mathbf{A} u, v\rangle_{\mathbb{H}} \text { for all } u \in D(\mathbf{A}), v \in D(\mathcal{E}) .
\end{aligned}
$$

Proof: Let $\alpha>0$ and $u \in D(\mathbf{A})$. Now $u \in G_{\alpha}(\mathbb{H}) \subset D(\mathcal{E})$ and we get for all $v \in D(\mathcal{E})$

$$
\begin{aligned}
\mathcal{E}(u, v) & =\mathcal{E}_{\alpha}\left(G_{\alpha} G_{\alpha}^{-1} u, v\right)-\alpha\langle u, v\rangle_{\mathbb{H}}=\left\langle G_{\alpha}^{-1} u, v\right\rangle_{\mathbb{H}}-\alpha\langle u, v\rangle_{\mathbb{H}} \\
& =\left\langle\left(G_{\alpha}^{-1}-\alpha\right) u, v\right\rangle_{\mathbb{H}}=\langle-\mathbf{A} u, v\rangle_{\mathbb{H}} .
\end{aligned}
$$

Theorem 3.4.8. Let $\left(G_{\alpha}\right)_{\alpha>0}$ be a continuous contraction resolvent on $\mathbb{H}$ with corresponding generator A. Define

$$
\mathcal{E}(u, v):=\langle-\mathbf{A} u, v\rangle_{\mathbb{H}}, \quad u, v \in D(\mathbf{A})
$$

Now $\left(G_{\alpha}\right)_{\alpha>0}$ and $(\mathcal{E}, D(\mathcal{E}))$ have the relation as in the above theorem.

These theorems state the relationship between the resolvent to a linear operator and its corresponding bilinear form.

\subsection{Dirichlet forms}

The theory of Dirichlet forms is used in the area of Markov process theory. Dirichlet forms are connected to potential theory and energy methods in contrast to partial differential equation theory which are the usual tools used in diffusion theory.

Definition 3.5.1. A coersive closed form $(\mathcal{E}, D(\mathcal{E}))$ on $L^{2}(E, m)$ is called a Dirichlet form if for all $u \in D(\mathcal{E})$ it holds

$$
\begin{aligned}
& \text { (i) } u^{+} \wedge 1 \in D(\mathcal{E}), \\
& \text { (ii) } \mathcal{E}\left(u+u^{+} \wedge 1, u-u^{+} \wedge 1\right) \geq 0, \\
& \text { (iii) } \mathcal{E}\left(u-u^{+} \wedge 1, u+u^{+} \wedge 1\right) \geq 0 .
\end{aligned}
$$

Remark 3.5.2. If $\mathcal{E}$ is symmetric a simple calculation shows that conditions (ii) and (iii) above are equivalent to

$$
\mathcal{E}\left(u^{+} \wedge 1, u^{+} \wedge 1\right) \leq \mathcal{E}(u, u)
$$


Definition 3.5.3. Let $\mathbb{H}=L^{2}(E, m)$. A bilinear form $\mathcal{E}$ on $L^{2}(E, m)$ is regular if $D(\mathcal{E}) \cap C_{0}(E)$ is dense in $D(\mathcal{E})$ w.r.t. $\mathcal{E}_{1}$-norm and dense in $C_{0}(E)$ w.r.t. the uniform norm. Here $C_{0}(E)$ denotes all continuous functions on $E$ with compact support.

We recall some basic definitions from the theory of stochastic processes.

Definition 3.5.4. We say that a function is cadlag if it is right continuous with left limits, i.e.

$$
\begin{array}{ll}
\text { (i) } f(x-):=\lim _{t \nearrow x} f(t) & \text { exists, } \\
\text { (ii) } f(x+):=\lim _{t \searrow x} f(t) & \text { exists and is equal to } f(x) \text {. }
\end{array}
$$

The corresponding term for left continuous functions is caglad.

Definition 3.5.5. Two stochastic processes $X$ and $Y$ with a common index set $T$ are called versions of one another if

$$
t \in T, P(\{\omega: X(t, \omega)=Y(t, \omega)\})=1 .
$$

Such processes are also said to be stochastically equivalent.

If in addition the process $X_{t}$ is left or right continuous then for a version $Y_{t}$ we have $X_{t}=Y_{t}$ almost surely.

It turns out that we only need to study the form on a dense subset of $D(\mathcal{E})$ and here the concept of nests appear.

Definition 3.5.6. (i) An increasing sequence $\left(F_{k}\right)_{k \in \mathbb{N}}$ of closed subsets of $E$ is called an $\mathcal{E}$-nest if $\cup_{k \geq 1} D(\mathcal{E})_{F_{k}}$ is dense in $D(\mathcal{E})$ w.r.t. $\|\cdot\|_{\tilde{\varepsilon}_{1}}$, where $D(\mathcal{E})_{F_{k}}$ denotes $\{u \in$ $D(\mathcal{E}): u=0 m$-a.e. on $\left.E \backslash F_{k}\right\}$.

(ii) A subset $N \subset \mathcal{E}$ is called $\mathcal{E}$-exceptional if $N \subset \cap_{k \geq 0} F_{k}^{c}$ for some $\mathcal{E}$-nest $\left(F_{k}\right)_{k \in \mathbb{N}}$. (iii) We say that a property holds $\mathcal{E}$-quasi-everywhere if it holds everywhere outside some $\mathcal{E}$-exceptional set.

We can relate the definition of quasi-continuity to a similar notion on the $\mathcal{E}$-nest.

Definition 3.5.7. An $\mathcal{E}$-quasi-everywhere defined function $f$ is called $\mathcal{E}$-quasi-continuous if there exists an $\mathcal{E}$-nest $\left(F_{k}\right)_{k \in \mathbb{N}}$ such that $f$ is continuous on $\left(F_{k}\right)_{k \in \mathbb{N}}$.

It is now possible to define what we mean with a quasi-regular Dirichlet form.

Definition 3.5.8. A Dirichlet form $(\mathcal{E}, D(\mathcal{E}))$ on $L^{2}(E, m)$ is called quasi-regular if:

(i) There exists an $\mathcal{E}$-nest $\left(F_{k}\right)_{k \in \mathbb{N}}$ consisting of compact sets,

(ii) There exists an $\tilde{\mathcal{E}}_{1}^{1 / 2}$-dense subset of $D(\mathcal{E})$ whose elements have $\mathcal{E}$-quasi-continuous $m$-versions,

(iii) There exist $u_{n} \in D(\mathcal{E}), n \in \mathbb{N}$, having $\mathcal{E}$-quasi-continuous $m$-versions $\tilde{u}_{n}$, $n \in \mathbb{N}$, and an $\mathcal{E}$-exeptional set $N \subset E$ such that $\left\{\tilde{u}_{n}: n \in \mathbb{N}\right\}$ separates the points of $E \backslash N$. 
In infinite dimension the quasi-regularity of the Dirichlet form is enough to be able to associate it with a special kind of process. The following definitions serve to clarify what we mean.

Definition 3.5.9. A process $M$ with state space $E$ is called $\mu$-tight if there exists an increasing sequence $\left(K_{n}\right)_{n \in \mathbb{N}}$ of compact sets in $E$ such that

$$
P_{\mu}\left(\lim _{n \rightarrow \infty} \inf \left\{t>0: M_{t} \in E \backslash K_{n}\right\}<\infty\right)=0
$$

where inf $\varnothing:=\infty$.

We note that it simply means that for every $\varepsilon>0$ there exists a compact set $K$ such that $P_{\mu}\left(M_{t} \in K\right)>1-\varepsilon$.

Definition 3.5.10. A cadlag Markov process $M$ with state space $E$ and transition semigroup $\left(p_{t}\right)_{t>0}$ is said to be properly associated with $(\mathcal{E}, D(\mathcal{E}))$ and its corresponding semigroup $\left(T_{t}\right)_{t>0}$ if $p_{t} f$ is an $\mathcal{E}$-quasi continuous $\mu$-version of $T_{t} f$ for all $t>0$ and all bounded $f \in L^{2}(E ; \mu)$.

The following theorem is the connection between the theory of forms and process theory.

Theorem 3.5.11. Let $(\mathcal{E}, D(\mathcal{E}))$ be a quasi-regular Dirichlet form on $L^{2}(E, \mu)$. Then there exists a pair $(M, M)$ of $\mu$-tight special standard processes which is properly associated with $(\mathcal{E}, D(\mathcal{E}))$.

Proof: The proof is lengthy and will therefore be omitted. The reader may find it in $[5]$.

Remark 3.5.12. It is known that if $E$ is locally compact then there exists an associated process to every regular Dirichlet form on $L^{2}(E, \mu)$. See [3].

The Cameron-Martin formula tells us how the Wiener measure changes under a translation. It was discovered by Robert Cameron and William Martin in 1944.

Theorem 3.5.13. Let $h \in \mathbb{H}$, where

$$
\mathbb{H}=\left\{f \in C([0,1]): f(0)=0, f \text { absolutely continuous, } \int_{0}^{1} f^{\prime}(u)^{2} d u<\infty\right\},
$$

and define $T_{h}(z):=z+h, z \in E$, where

$$
E=\{f \in C([0,1]): f(0)=0\} .
$$

Let $\mu \circ T_{h}^{-1}$ denote the image measure of $\mu$ under $T_{h}$. Then

$$
\frac{d \mu \circ T_{h}^{-1}}{d \mu}=\exp \left\{W_{h}-\frac{1}{2}\|h\|_{\mathbb{H}}^{2}\right\} .
$$


Proof: Let $f, h \in \mathbb{H}$ and $\alpha:=\langle f, h\rangle_{\mathbb{H}} /\langle h, h\rangle_{\mathbb{H}}$. Then $W_{h}$ and $W_{f-\alpha h}$ are independent since they are Gaussian and

$$
E\left[W_{h} W_{f-\alpha h}\right]=\langle h, f-\alpha h\rangle_{\mathbb{H}}=\langle h, f\rangle_{\mathbb{H}}-\langle f, h\rangle_{\mathbb{H}}=0 .
$$

We have

$$
\begin{aligned}
\int & \exp \left\{i\langle f, z\rangle_{\mathbb{H}}\right\} \exp \left\{W_{h}(z)-\frac{1}{2}\|h\|_{\mathbb{H}}^{2}\right\} \mu(d z) \\
= & \exp \left\{-\frac{1}{2}\|h\|_{\mathbb{H}}^{2}\right\} \int \exp \left\{i\langle f, z\rangle_{\mathbb{H}}+\langle h, z\rangle_{\mathbb{H}}\right\} d \mu \\
= & \exp \left\{-\frac{1}{2}\|h\|_{\mathbb{H}}^{2}\right\} \int \exp \left\{i\langle f-\alpha h, z\rangle_{\mathbb{H}}+\langle(1+i \alpha) h, z\rangle_{\mathbb{H}}\right\} d \mu \\
= & \exp \left\{-\frac{1}{2}\|h\|_{\mathbb{H}}^{2}\right\} \int \exp \left\{i W_{f-\alpha h}+(1+i \alpha) W_{h}\right\} d \mu \\
= & \exp \left\{-\frac{1}{2}\|h\|_{\mathbb{H}}^{2}\right\} \int \exp \left\{i W_{f-\alpha h}\right\} d \mu \int \exp \left\{i(-i+\alpha) W_{h}\right\} d \mu \\
= & \exp \left\{-\frac{1}{2}\|h\|_{\mathbb{H}}^{2}\right\} \exp \left\{-\frac{1}{2}\|f-\alpha h\|_{\mathbb{H}}^{2}\right\} \exp \left\{-\frac{1}{2}(-i+\alpha)^{2}\|h\|_{\mathbb{H}}^{2}\right\} \\
= & \exp \left\{-\frac{1}{2}\|h\|_{\mathbb{H}}^{2}-\frac{1}{2}\|f-\alpha h\|_{\mathbb{H}}^{2}+\frac{1}{2}\|h\|_{\mathbb{H}}^{2}+i \alpha\|h\|_{\mathbb{H}}^{2}-\frac{1}{2} \alpha^{2}\|h\|_{\mathbb{H}}^{2}\right\} \\
= & \exp \left\{-\frac{1}{2}\langle f, f\rangle_{\mathbb{H}}+i\langle f, h\rangle_{\mathbb{H}}\right\} \\
= & \int \exp \left\{i\langle f, z\rangle_{\mathbb{H}}\right\}\left(\mu \circ T_{h}^{-1}\right)(d z) .
\end{aligned}
$$

Keeping all these definitions and theorems in mind we are now well equipped to study the Dirichlet form in the next chapter. 



\section{4}

Chapter 4-Main results

$\mathrm{T}$

HE paper included here is a joint work with Jörg-Uwe Löbus and is the result of a study at the department of Mathematical Statistics at Linköping University during 2012. This version of the paper contains slightly more details than the version available at ArXiv and the version submitted for publication. The aim of the paper is to take results from the finite dimensional theory into the infinite dimensional case. This is done via the means of a coordinate representation.

\subsection{Earlier work}

In the paper Construction of diffusions on path and loop spaces of compact Riemannian manifolds [2] the idea of Dirichlet form theory is used to create a diffusion process. More precisely the idea is that every quasi-regular Dirichlet form has an associated diffusion process. In [2] a form defined on a compact Riemannian manifold with these properties is presented.

The paper A class of processes on the path space over a compact riemannian manifold with unbounded diffusion [4] expands on the same idea. Here the form is

$$
\int\langle D F, D A G\rangle_{\mathbb{H}} d \nu
$$

where the operator $A$ is defined by

$$
A \Phi(\gamma):=\sum_{i=1}^{\infty} \lambda_{i}\left\langle S_{i}, \Phi(\gamma)\right\rangle_{\mathbb{H}} S_{i}, \Phi \in D(A),
$$

and the diffusion coefficients $\lambda$ are assumed to be bounded from below and possibly unbounded. It is shown that under the assumption $\lambda_{n} \leq c n^{1-\varepsilon}$, the form is a quasiregular Dirichlet form and thus has an associated process. 
The paper presented in this thesis is similar to the paper Quasi-regular Dirichlet forms on Riemannian path and loop spaces [11]. Both these papers investigate infinite dimensional diffusion processes where the diffusion is related to possibly unbounded operators. Both papers refer to the ideas of [4]. Let us summarize the differences between this paper and [11]. The main difference is that the theory in [11] is presented in a geometric setting, i.e. on a manifold while here we study the flat case. Even though the geometric framework in [11] is more general, our coordinatewise non-geometric setting makes it easier to focus on the phenomenon we are interested in to investigate, namely pointwise unboundedness of diffusion. The forms studied in these two papers are of type (4.1). The form in [11] differs from the one presented here in the sense that the operator $A$ in this paper is defined pointwise, constant over the trajectory space, while in [11] it can vary, i.e. $A=A(\gamma)$. The operator in [11] is defined by letting $(A, D(A))$ be a densely defined self-adjoint operator on $L^{2}\left(W_{0} \rightarrow \mathbb{H} ; \mu\right)$ such that

$$
\begin{aligned}
& A f \Phi=f A \Phi \text { for } f \in L^{\infty}(\mu) \text { and } \Phi \in L^{2}\left(W_{0} \rightarrow \mathbb{H} ; \mu\right), \\
& \text { and } A \geq \varepsilon I \text { for some } \varepsilon>0 .
\end{aligned}
$$

Here $W_{0}$ is a manifold and $I$ denotes the identity operator. They define

$$
\mathcal{E}_{A}(F, G)=\int_{W_{0}}\left\langle A^{1 / 2} D F, A^{1 / 2} D G\right\rangle_{\mathbb{H}} d \mu, \quad F, g \in Y,
$$

where

$$
Y:=\left\{F \in \mathcal{F} C_{b}^{\infty}: D F \in D\left(A^{1 / 2}\right)\right\},
$$

and $\mathcal{F} C_{b}^{\infty}$ denotes the set of cylindrical functions $\left\{F(\gamma)=f\left(\gamma\left(s_{1}\right), \ldots, \gamma\left(s_{k}\right)\right): s_{j} \in\right.$ $\left.[0,1], f \in C_{b}^{\infty}\right\}$. In addition the assumption

$$
\left\|A^{1 / 2} \Phi_{t, v}\right\|_{L^{2}\left(W_{0} \rightarrow \mathbb{H} ; \mu\right)}^{2} \leq C\|v\|_{L^{\infty}\left(W_{0} \rightarrow \mathbf{R}^{d} ; \mu\right)}^{2}, t \in[0, T], v \in L^{\infty}\left(W_{0} \rightarrow \mathbf{R}^{d} ; \mu\right),
$$

is made. In our situation with $A$ constant on the trajectory space and with non-decreasing eigenvalues, the conditions (A0) and (A1) are satisfied. In [11] the condition

$$
\sum_{j=1}^{d} \mu\left(\lambda_{i}\right)+\sum_{m=0}^{\infty} \sum_{j=1}^{d} \max _{1 \leq k \leq 2^{m}} \mu\left(\lambda_{d\left(2^{m}+k-1\right)+j}\right) 2^{-m}<\infty
$$

is shown to be sufficient for the closability of the form. In our case the condition is reduced to

$$
\sum_{p=0}^{\infty} \frac{\lambda_{d 2^{p}}}{2^{p}}<\infty
$$

a relation first noted in [4]. Moreover we show that this condition is necessary in the sense of Proposition 4.5.6 (c). In order to handle weights with respect to the Wiener measure [11] introduces geometric conditions (1.3), (1.5), and (1.6). We use the condition (4.12) in Lemma 4.5.3 which is compatible with [2]. To show quasi-regularity both this paper and [11] use the method of [2], [4], and [9]. However, the proof in [11] involves a (geometric) result of [10]. In contrast, our proof uses Doob's inequality in that particular point of the proof. 


\subsection{Introduction}

This paper is concerned with Dirichlet forms of type

$$
\mathcal{E}(F, G)=\int\langle D F, A D G\rangle_{\mathbb{H}} \varphi d \nu=\int\left\langle D F, \sum_{i=1}^{\infty} \lambda_{i}\left\langle S_{i}, D G\right\rangle_{\mathbb{H}} S_{i}\right\rangle_{\mathbb{H}} \varphi d \nu,
$$

where the diffusion operator $A$ is in general unbounded, cf. [4]. We are interested in weight functions $\varphi$ of the form

$$
\varphi(\gamma)=\exp \left\{\int_{0}^{1}\left\langle b\left(\gamma_{s}\right), d \gamma_{s}\right\rangle_{\mathbb{R}^{d}}-\frac{1}{2} \int_{0}^{1}\left\|b\left(\gamma_{s}\right)\right\|^{2} d s\right\} .
$$

This choice of weight functions is motivated by the papers $[11,12]$ by F.-Y. Wang and $\mathrm{B}$. $\mathrm{Wu}$. In this way it is possible to relate and compare the results of the present paper to the findings there. We present our ideas in terms of infinite dimensional processes on the classical Wiener space using a coordinate representation. This makes the subject comprehensible, in particular, to readers familiar with the finite dimensional theory. The form is studied on the set of smooth cylindrical functions

$$
F, G \in Y=\left\{F(\gamma)=f\left(\gamma\left(s_{1}\right), \ldots, \gamma\left(s_{k}\right)\right): s_{j} \text { is a dyadic point }\right\},
$$

where $\gamma$ is a Wiener trajectory. We do this as well on the more common set of cylindrical functions

$$
F, G \in Z=\left\{F(\gamma)=f\left(\gamma\left(s_{1}\right), \ldots, \gamma\left(s_{k}\right)\right): s_{j} \in[0,1]\right\} .
$$

Well-definiteness of $\mathcal{E}$ on $Y$ is a consequence of the fact that the sum in (4.2) is finite. Well-definiteness of $\mathcal{E}$ on $Z$ requires the convergence of the sum in (4.2). These two different initial situations result in possibly different closures of $(\mathcal{E}, Y)$ and $(\mathcal{E}, Z)$ on $L^{2}(\varphi \nu)$. Using the coordinate representation in (4.2) we give conditions for closability. The requirement of $\varphi^{-1} \in L^{1}(\nu)$ would be sufficient, for example, for closability in the classical case with bounded cylindrical functions and $\lambda_{1}=\lambda_{2}=\ldots=1$ on $L^{2}(\nu)$, see [5]. However, the paper investigates forms of the structure (4.2) with an in general unbounded diffusion operator. We give necessary and sufficient conditions on the increase of $\lambda_{1}, \lambda_{2}, \ldots$ that guarantee closability of $(\mathcal{E}, Y)$ and $(\mathcal{E}, Z)$ on $L^{2}(\varphi \nu)$ in terms of the Schauder functions $S_{i}, i \in \mathbb{N}$, the coordinate functions in $\mathbb{H}$. Locality, Dirichlet property, and quasi-regularity of the closure $(\mathcal{E}, Z)$ on $L^{2}(\varphi \nu)$ is then obtained by using methods of $[1,2,4,5,9]$. We are also interested in characterizing the associated process in terms of their local first and second moments of the form

$$
\lim _{t \rightarrow 0} \frac{1}{t} \int\left(\mathbf{S}_{i}(\gamma)-\mathbf{S}_{i}(\tau)\right) P_{\tau}\left(X_{t} \in d \gamma\right)
$$

and

$$
\lim _{t \rightarrow 0} \frac{1}{t} \int\left(\mathbf{S}_{i}(\gamma)-\mathbf{S}_{i}(\tau)\right)^{2} P_{\tau}\left(X_{t} \in d \gamma\right),
$$

where $\mathbf{S}_{i}$ are certain linear functions on the trajectory space. We represent these local moments in terms of the sequence $\lambda_{1}, \lambda_{2}, \ldots$ and the weight function $\varphi$. Corresponding to the closability condition of $(\mathcal{E}, Z)$ on $L^{2}(\varphi \nu)$, we derive a necessary and sufficient condition for the limits (4.3) and (4.4) to exist. This way lets us obtain compatibility with the classical Kolmogorov characterization of finite dimensional diffusion processes. 


\subsection{Formal definitions}

We study the form on the space $L^{2}(\varphi \nu) \equiv L^{2}(\Omega, \varphi \nu)$ where $\Omega:=C_{0}\left([0,1] ; \mathbb{R}^{d}\right):=$ $\left\{f \in C\left([0,1] ; \mathbb{R}^{d}, f(0)=0\right\}, \nu\right.$ is the Wiener measure on $\Omega$ and $\varphi$ is a density function specified below. As stated earlier, the form is given by

$$
\mathcal{E}(F, G)=\int\langle D F, A D G\rangle_{\mathbb{H}} \varphi d \nu, \quad F, G \in D(\mathcal{E}),
$$

where $\mathbb{H}$ is the Cameron-Martin space, i.e., the space of all absolutely continuous $\mathbb{R}^{d}$ valued functions $f$ on $[0,1]$, with $f(0)=0$ and equipped with inner product

$$
\langle\varphi, \psi\rangle_{\mathbb{H}}:=\int_{[0,1]}\left\langle\varphi^{\prime}(x), \psi^{\prime}(x)\right\rangle_{\mathbb{R}^{d}} d x .
$$

Motivated by [11], we suppose in sections 4.7 and 4.8 that $\varphi: \Omega \rightarrow[0, \infty]$ has the form

$$
\varphi(\gamma)=\exp \left\{\int_{0}^{1}\left\langle b\left(\gamma_{s}\right), d \gamma_{s}\right\rangle_{\mathbb{R}^{d}}-\frac{1}{2} \int_{0}^{1}\left\|b\left(\gamma_{s}\right)\right\|_{\mathbb{R}^{d}}^{2} d s\right\}
$$

where $b: \mathbb{R}^{d} \rightarrow \mathbb{R}^{d}$ is the gradient of a function $f \in C_{b}^{2}\left(\mathbb{R}^{d} ; \mathbb{R}\right)$. Then by Itô's formula, $\varphi$ defined by (4.6) is both bounded from below and from above, on $\Omega$. We define the set of all cylindrical functions

$$
\begin{aligned}
Z:= & \left\{F(\gamma)=f\left(\gamma\left(s_{1}\right), \ldots, \gamma\left(s_{k}\right)\right), \gamma \in \Omega:\right. \\
& \left.0<s_{1}<\cdots<s_{k}=1, f \in C_{p}^{\infty}\left(\mathbb{R}^{d^{k}}\right), k \in \mathbb{N}\right\}
\end{aligned}
$$

where $C_{p}^{\infty}$ denotes smooth functions with polynomial growth. We also define

$$
\begin{aligned}
Y:= & \left\{F(\gamma)=f\left(\gamma\left(s_{1}\right), \ldots, \gamma\left(s_{k}\right)\right), \gamma \in \Omega:\right. \\
& \left.F \in Z, s_{1}, \ldots, s_{k} \in\left\{\frac{l}{2^{n}}: l \in\left\{1, \ldots, 2^{n}\right\}\right\}, n \in \mathbb{N}\right\} .
\end{aligned}
$$

For $F \in Z$ and $\gamma \in \Omega$ the gradient operator $D$ is defined by

$$
D_{s} F(\gamma)=\sum_{i=1}^{k}\left(s_{i} \wedge s\right)\left(\nabla_{s_{i}} f\right)(\gamma), \quad s \in[0,1],
$$

where $\left(\nabla_{s_{i}} f\right)(\gamma)=\left(\nabla_{s_{i}} f\right)\left(\gamma\left(s_{1}\right), \ldots, \gamma\left(s_{k}\right)\right)$ denotes the gradient of the function $f$ relative to the $i$ th variable while holding the other variables fixed. We let $\left(e_{j}\right)_{j=1, \ldots, d}$ denote the standard basis in $\mathbb{R}^{d}$ and

$$
\begin{aligned}
& H_{1}(t)=1, \quad t \in[0,1], \\
& H_{2^{m}+k}(t)=\left\{\begin{array}{ll}
2^{m / 2} & \text { if } t \in\left[\frac{k-1}{2^{m}}, \frac{2 k-1}{2^{m+1}}\right) \\
-2^{m / 2} & \text { if } t \in\left[\frac{2 k-1}{2^{m+1}}, \frac{k}{2^{m}}\right) \\
0 & \text { otherwise }
\end{array} \quad k=1, \ldots, 2^{m}, m=0,1, \ldots,\right.
\end{aligned}
$$


denote the system of the Haar functions on $[0,1]$. We also define

$$
g_{d(r-1)+j}:=H_{r} \cdot e_{j}, \quad r \in \mathbb{N}, j \in\{1, \ldots, d\},
$$

and

$$
S_{n}(s):=\int_{0}^{s} g_{n}(u) d u, \quad s \in[0,1], n \in \mathbb{N}
$$

\subsection{Definition of the form}

We use the following definitions from [4]. We choose a non-decreasing sequence of positive numbers $\lambda_{1}, \lambda_{2}, \ldots$

$$
\begin{gathered}
D(A):=\left\{\Phi \in L^{2}(\Omega \rightarrow \mathbb{H}, \nu): \int \sum_{i=1}^{\infty} \lambda_{i}^{2}\left\langle S_{i}, \Phi\right\rangle_{\mathbb{H}}^{2} d \nu<\infty\right\}, \\
A \Phi(\gamma):=\sum_{i=1}^{\infty} \lambda_{i}\left\langle S_{i}, \Phi(\gamma)\right\rangle_{\mathbb{H}} S_{i}, \quad \gamma \in \Omega, \quad \Phi \in D(A) .
\end{gathered}
$$

We can then conclude that

$$
\begin{aligned}
\mathcal{E}(F, F) & =\int\langle D F, A D F\rangle_{\mathbb{H}} \varphi d \nu=\int\left\langle D F, \sum_{i=1}^{\infty} \lambda_{i}\left\langle S_{i}, D F\right\rangle_{\mathbb{H}} S_{i}\right\rangle_{\mathbb{H}} \varphi d \nu \\
& =\sum_{i=1}^{\infty} \lambda_{i} \int\left\langle S_{i}, D F\right\rangle_{\mathbb{H}}^{2} \varphi d \nu<\infty, \quad F \in Y,
\end{aligned}
$$

is well defined since, for $F \in Y$, this is just a finite sum.

\subsection{Closability}

In this section we first prove a general closability result for the Malliavin gradient. Using this result, a criterion for the closability of the form is formulated. We note that, under these conditions, the form is local. We assume that $\varphi \leq c<\infty$.

Lemma 4.5.1. If $x_{n} \rightarrow x$ in $L^{2}(\varphi \nu ; \mathbb{H})$ where $\mathbb{H}=L^{2}(T), T=[0,1]$, and

$$
\lim _{n \rightarrow \infty} \int \psi \delta\left(x_{n}\right) d \nu=0
$$

for all $\psi$ in $Z$, then $x=0$.

Proof: For any $\phi \in \mathcal{H}_{m+1}$, we have $\phi=I_{m+1}(g)$ for some symmetric $g \in L^{2}\left(T^{m+1}\right)$. We have for all $n \in \mathbb{N}$

$$
x_{n}(t)=\sum_{j=0}^{\infty} I_{j}\left(f_{j}^{(n)}(\cdot, t)\right)
$$


where $f_{j}^{(n)} \in L^{2}\left(T^{j+1}\right)$ where for $j \geq 1, f_{j}^{(n)}$ is symmetric in the first $j$ variables. Thus

$$
\delta\left(x_{n}\right)=\sum_{j=0}^{\infty} I_{j+1}\left(\tilde{f}_{j}^{(n)}\right) .
$$

We can restrict ourselves to $\phi \in \mathcal{H}_{m+1}$ such that $\phi \in Z$. By hypothesis we have

$$
\begin{gathered}
(m+1) !\left\langle g, \tilde{f}_{m}^{(n)}\right\rangle_{L^{2}\left(T^{m+1}\right)}=\int I_{m+1}(g) I_{m+1}\left(f_{m}^{(n)}\right) d \nu \\
=\int \phi \delta\left(x_{n}\right) d \nu \rightarrow 0, \text { as } n \rightarrow \infty .
\end{gathered}
$$

Since $g \in L^{2}\left(T^{m+1}\right)$ is symmetric we have

$$
\left\langle g, f_{m}^{(n)}\right\rangle_{L^{2}\left(T^{m+1}\right)} \rightarrow 0, \text { as } n \rightarrow \infty .
$$

For all symmetric $h \in L^{2}\left(T^{m}, \mathbb{H}\right)$ we obtain

$$
\left\langle h, f_{m}^{(n)}(\cdot, t)\right\rangle_{L^{2}\left(T^{m} ; \mathbb{H}\right)} \rightarrow 0, \text { as } n \rightarrow \infty,
$$

where the letter $t$ indicates the variable for the function in $\mathbb{H}$. Using the fact that the $m$ th Wiener chaos is isometric to the space of symmetric functions $L_{s}^{2}\left(T^{m}\right)$ we get for any $\psi \in L^{2}(\nu ; \mathbb{H})$ such that $\psi(\cdot, t) \in \mathcal{H}_{m}$ for a.e. $t \in[0,1], \psi=I_{m}(h)$, we have

$$
\left\langle I_{m}(h), I_{m}\left(f_{m}^{(n)}(\cdot, t)\right)\right\rangle_{L^{2}(\nu ; \mathbb{H})} \rightarrow 0, \text { as } n \rightarrow \infty .
$$

Since $I_{m}\left(f_{m}^{(n)}(\cdot, t)\right)$ is the projection of $x_{n}$ to the $m$ th Wiener chaos we have

$$
\left\langle x_{n}, \tilde{\phi}\right\rangle_{L^{2}(\nu ; \mathbb{H})} \underset{n \rightarrow \infty}{\longrightarrow} 0
$$

for all finite linear combinations $\tilde{\phi}=\alpha_{1} \psi_{1}+\ldots+\alpha_{k} \psi_{k}$, where $\psi_{i} \in L^{2}(\nu ; \mathbb{H}), \psi_{i}(\cdot, t)$ is from the $i$ th Wiener chaos for a.e. $t \in[0,1]$, and $i \in\{1, \ldots, k\}$. Since $\varphi$ is bounded this implies

$$
\left\langle x_{n}, \tilde{\phi}\right\rangle_{L^{2}(\varphi \nu ; \mathbb{H})} \underset{n \rightarrow \infty}{\longrightarrow} 0 .
$$

Since also $\left\|x_{n}\right\|_{L^{2}(\varphi \nu ; \mathbb{H})}$ is bounded we have

$$
\left\langle x_{n}, \tilde{\phi}\right\rangle_{L^{2}(\varphi \nu ; \mathbb{H})} \underset{n \rightarrow \infty}{\longrightarrow} 0, \quad \text { for all } \tilde{\phi} \in L^{2}(\varphi \nu ; \mathbb{H}) .
$$

This contradicts $x_{n} \rightarrow x$ in $L^{2}(\varphi \nu ; \mathbb{H})$ and the statement follows.

Lemma 4.5.2. If

$$
\frac{f}{\varphi} \in L^{1}(\nu), \quad \forall f \in Z,
$$

then $\left\{\frac{1}{\varphi} D \psi: \psi \in Z\right\}$ is a dense subset of $L^{2}(\varphi \nu ; \mathbb{H})$. 
Proof: We assume the contrary. Then we can find $x \in L^{2}(\varphi \nu ; \mathbb{H})$ and cylindrical functions $x_{n}, n \in \mathbb{N}$, with values in $\mathbb{H}$, such that $x \neq 0$ and $x_{n} \underset{n \rightarrow \infty}{\longrightarrow} x$ in $L^{2}(\varphi \nu ; \mathbb{H})$ and

$$
\int \frac{1}{\varphi}\langle D \psi, x\rangle_{\mathbb{H}} \varphi d \nu=0
$$

for all $\psi \in Z$. It follows that

$$
0=\int \frac{1}{\varphi}\langle D \psi, x\rangle_{\mathbb{H}} \varphi d \nu=\lim _{n \rightarrow \infty} \int\left\langle D \psi, x_{n}\right\rangle_{\mathbb{H}} d \nu=\lim _{n \rightarrow \infty} \int \psi \delta\left(x_{n}\right) d \nu
$$

for all $\psi \in Z$. From this we get $x=0$ by Lemma 4.5.1, and we have a contradiction.

Lemma 4.5.3. If $0<\varphi \leq c$ for some $c \in \mathbb{R}_{+}$, and (4.12) holds, then $(\mathscr{D}, Z)$ defined by

$$
\mathscr{D}(f, g):=\frac{1}{2} \int\langle D f, D g\rangle_{\mathbb{H}} \varphi d \nu, \quad f, g \in Z
$$

is closable on $L^{2}(\varphi \nu)$. Let $L$ denote the generator of $\mathscr{D}$ and note that $L$ is the OrnsteinUhlenbeck operator.

Proof: Let $u_{n} \in Z, \psi \in Z$ and

$$
u_{n} \underset{n \rightarrow \infty}{\longrightarrow} 0 \text { in } L^{2}(\varphi \nu), \quad D u_{n} \underset{n \rightarrow \infty}{\longrightarrow} f \quad \text { in } L^{2}(\varphi \nu ; \mathbb{H})
$$

Then

$$
\begin{aligned}
\frac{1}{2} \int\left\langle D u_{n}, \frac{1}{\varphi} D \psi\right\rangle_{\mathbb{H}} \varphi d \nu & =\frac{1}{2} \int\left\langle D u_{n}, D \psi\right\rangle_{\mathbb{H}} d \nu \\
& =\int u_{n}(-L \psi) d \nu \\
& =\int u_{n} \frac{1}{\varphi}(-L \psi) \varphi d \nu
\end{aligned}
$$

By assumption we have $1 / \varphi \cdot(-L \psi)^{2} \in L^{1}(\nu)$, i.e. $1 / \varphi \cdot(-L \psi) \in L^{2}(\varphi \nu)$. Thus

$$
\int u_{n} \frac{1}{\varphi}(-L \psi) \varphi d \nu \underset{n \rightarrow \infty}{\longrightarrow} 0 .
$$

Since Lemma 4.5.2 showed that $\left\{\frac{1}{\varphi} D \psi: \psi \in Z\right\}$ is a dense subset of $L^{2}(\varphi \nu ; \mathbb{H})$, it now follows that $f=0$. Hence the form is closable.

Remark 4.5.4. The closability condition (4.12) would weaken to $1 / \varphi \in L^{1}(\nu)$ if the initial definition of $\mathscr{D}$ was on all bounded $f, g \in Z$. We get compability with [5], section II.2 a).

We also formulate the following lemma that serves an important role in proving and formulating the closability conditions of the form. 
Lemma 4.5.5. (a) If $s_{k}=\sum_{i=1}^{r} c_{i} \cdot 2^{-i}, i_{p, j}=2^{p-1}(d+j-1)+1+\sum_{q=0}^{p-1} c_{q} 2^{p-q-1}$ and $j \in\{1, \ldots, d\}$ where $c_{1}, \ldots, c_{r-1} \in\{0,1\}, c_{r}=1, c_{0}=c_{r+1}=0$ and $r \geq 2$, then

$$
\sum_{i=1}^{\infty} \lambda_{i}\left\langle S_{i}\left(s_{k}\right), e_{j}\right\rangle_{\mathbb{R}^{d}}^{2}=\lambda_{j} s_{k}^{2}+\sum_{p=1}^{r} \lambda_{i_{p, j}} 2^{p-1}\left(c_{p} 2^{-p}+(-1)^{c_{p}} \sum_{q=p+1}^{r+1} 2^{-q} c_{q}\right)^{2} .
$$

(b) The relation

$$
\sup _{\substack{c_{1}, \ldots, c_{r-1} \in\{0,1\}, c_{r}=1, c_{r+1}=0, j \in\{1, \ldots, d\}, r \geq 2}} \sum_{p=1}^{r} \lambda_{i_{p, j}} 2^{p-1}\left(c_{p} 2^{-p}+(-1)^{c_{p}} \sum_{q=p+1}^{r+1} 2^{-q} c_{q}\right)^{2}<\infty
$$

is equivalent to

$$
\sum_{p=0}^{\infty} \frac{\lambda_{d 2^{p}}}{2^{p}}<\infty
$$

Proof: a) Since $e_{j} \perp S_{i}$ for $i \bmod d \neq j$ and $S_{i}\left(s_{k}\right)=0$ for $i>d 2^{r}$ we get

$$
\sum_{i=1}^{\infty} \lambda_{i}\left(S_{i}\left(s_{k}\right) \cdot e_{j}\right)^{2}=\lambda_{j}\left(S_{j}\left(s_{k}\right) \cdot e_{j}\right)^{2}+\sum_{i=1}^{2^{r}} \lambda_{d i+j}\left(S_{d i+j}\left(s_{k}\right) \cdot e_{j}\right)^{2} .
$$

Now since

$$
\lambda_{j}\left(S_{j}\left(s_{k}\right) \cdot e_{j}\right)^{2}=\lambda_{j} s_{k}^{2}
$$

and

$$
S_{d i+j}\left(s_{k}\right)=0
$$

unless $d i+j=2^{p-1}(d+j-1)+1+\sum_{q=0}^{p-1} c_{q} 2^{p-q-1}$ for some $p \geq 1$. We get

$$
\begin{aligned}
& \left.\sum_{i=1}^{2^{r}} \lambda_{d i+j}\left(S_{d i+j}\left(s_{k}\right)\right) \cdot e_{j}\right)^{2} \\
& \quad=\sum_{p=1}^{r} \lambda_{i_{p, j}}\left(S_{i_{p, j}}\left(s_{k}\right) \cdot e_{j}\right)^{2},
\end{aligned}
$$

where

$$
\begin{aligned}
S_{i_{p, j}}\left(s_{k}\right) \cdot e_{j} & = \begin{cases}2^{\frac{p-1}{2}} \sum_{q=p+1}^{r+1} 2^{-q} c_{q} & \text { if } c_{p}=0 \\
2^{-\frac{p+1}{2}}-2^{\frac{p-1}{2}} \sum_{q=p+1}^{r+1} 2^{-q} c_{q} & \text { if } c_{p}=1\end{cases} \\
& =2^{\frac{p-1}{2}}\left(c_{p} 2^{-p}+(-1)^{c_{p}} \sum_{q=p+1}^{r+1} 2^{-q} c_{q}\right),
\end{aligned}
$$

and thus the statement follows.

(b) Assuming (4.13). Recall $\lambda_{1}, \lambda_{2}, \ldots$ is nondecreasing and thus $\lambda_{d 2^{p-1}}<\lambda_{i_{p, j}} \leq \lambda_{d 2^{p}}$. 
Using $c_{1}=c_{3}=c_{5}=\cdots=1, c_{2}=c_{4}=c_{6}=\cdots=0$ we get

$$
\begin{gathered}
\sup _{\substack{c_{1}, \ldots, c_{r-1} \in\{0,1\}, c_{r}=1, c_{r+1}=0, r \geq 2}} \sum_{p=1}^{r} \lambda_{i_{p, j}} 2^{p-1}\left(c_{p} 2^{-p}+(-1)^{c_{p}} \sum_{q=p+1}^{r+1} 2^{-q} c_{q}\right)^{2} \\
\geq \sum_{p=1}^{\infty} \lambda_{i_{p, j}} 2^{p-1}\left(2^{-p-1}\right)^{2}=\frac{1}{8} \sum_{p=1}^{\infty} \frac{\lambda_{i_{p, j}}}{2^{p}} \\
\geq \frac{1}{8} \sum_{p=1}^{\infty} \frac{\lambda_{d 2^{p-1}}}{2^{p}}=\frac{1}{16} \sum_{p=0}^{\infty} \frac{\lambda_{d 2^{p}}}{2^{p}} .
\end{gathered}
$$

Assuming (4.14) we obtain

$$
\begin{aligned}
\sup _{\substack{c_{1}, \ldots, c_{r-1} \in\{0,1\} \\
c_{r}=1, c_{r+1}=0, r \geq 2}} \sum_{p=1}^{r} \lambda_{i_{p, j}} 2^{p-1}\left(c_{p} 2^{-p}+(-1)^{c_{p}} \sum_{q=p+1}^{r+1} 2^{-q} c_{q}\right)^{2} \\
\leq \sum_{p=1}^{\infty} \lambda_{i_{p, j}} 2^{p-1}\left(2^{-p}\right)^{2}=\frac{1}{2} \sum_{p=1}^{\infty} \frac{\lambda_{i_{p, j}}}{2^{p}} \leq \frac{1}{2} \sum_{p=1}^{\infty} \frac{\lambda_{d 2^{p}}}{2^{p}} .
\end{aligned}
$$

The statement now follows.

Proposition 4.5.6. Let $\varphi$ satisfy (4.12).

(a) The form $(\mathcal{E}, Y)$ is closable in $L^{2}(\varphi \nu)$. Let $\left(\mathcal{E}, D_{Y}(\mathcal{E})\right)$ denote the closure of $(\mathcal{E}, Y)$ in $L^{2}(\varphi \nu)$.

(b) If

$$
\sum_{p=0}^{\infty} \frac{\lambda_{d 2^{p}}}{2^{p}}<\infty
$$

then $(\mathcal{E}, Z)$ is closable and we let $\left(\mathcal{E}, D_{Z}(\mathcal{E})\right)$ denote the closure of $(\mathcal{E}, Z)$ on $L^{2}(\varphi \nu)$. Furthermore $D_{Z}(\mathcal{E})=D_{Y}(\mathcal{E})$ under (4.15).

(c) $Z \subset D_{Y}(\mathcal{E})$ if and only if (4.15).

Proof: (a) We have

$$
\mathcal{E}(F, F)=\sum_{i=1}^{\infty} \lambda_{i} \int\left\langle S_{i}, D F\right\rangle_{\mathbb{H}}^{2} \varphi d \nu=\int\left\langle A^{1 / 2} D F, A^{1 / 2} D F\right\rangle_{\mathbb{H}} \varphi d \nu
$$

Suppose $\left\{F_{n}\right\}_{n \geq 1} \in Y$ such that $F_{n} \underset{n \rightarrow \infty}{\longrightarrow} 0$ in $L^{2}(\varphi \nu)$ and $\mathcal{E}\left(F_{n}-F_{m}, F_{n}-F_{m}\right) \rightarrow 0$. Since it follows that $A^{1 / 2} D F_{n}$ is Cauchy in $L^{2}(\Omega \rightarrow \mathbb{H}, \varphi \nu)$ we may define

$$
\psi:=\lim _{n \rightarrow \infty} A^{1 / 2} D F_{n}
$$

We also define

$$
J h:=\sum_{i=1}^{\infty} \lambda_{i}^{-1 / 2}\left\langle S_{i}, h\right\rangle_{\mathbb{H}} S_{i}
$$


The operator $J$ is bounded in $L^{2}(\Omega \rightarrow \mathbb{H}, \varphi \nu)$ and it follows

$$
D F_{n}=J A^{1 / 2} D F_{n} \underset{n \rightarrow \infty}{\longrightarrow} J \psi \text { in } L^{2}(\Omega \rightarrow \mathbb{H}, \varphi \nu) .
$$

From Lemma 4.5.3, it is known that $(D, Z)$ is closable on $L^{2}(\varphi \nu)$. It follows that $D F_{n} \underset{n \rightarrow \infty}{\longrightarrow} 0$ and thus $J \psi=0$. Since $\lambda_{i}>0$ and $\lambda_{i}^{-1 / 2}>0$, (4.16) gives $\psi=0$. Now $A^{1 / 2} D F_{n} \underset{n \rightarrow \infty}{\longrightarrow} 0$ and thus $\mathcal{E}\left(F_{n}, F_{n}\right)=\int\left\langle A^{1 / 2} D F_{n}, A^{1 / 2} D F_{n}\right\rangle_{\mathbb{H}} \varphi d \nu \rightarrow 0$ as $n \rightarrow \infty$.

(b) We show that (4.15) implies $Z \subset D_{Y}(\mathcal{E})$. Then $Y \subset Z \subset D_{Y}(\mathcal{E})$. Since $(\mathcal{E}, Y)$ is closable with closure $\left(\mathcal{E}, D_{Y}(\mathcal{E})\right)$, cf. (a), $(\mathcal{E}, Z)$ is then also closable and has $\left(\mathcal{E}, D_{Y}(\mathcal{E})\right)$ as its closure i.e. $D_{Y}(\mathcal{E})=D_{Z}(\mathcal{E})$.

Let $x^{v}(p)$ denote the $v$ th coordinate of $p \in \mathbb{R}^{d}, v \in\{1, \ldots, d\}$. Let us demonstrate that $F(\gamma)=x^{v}(\gamma(s)) \in D_{Y}(\mathcal{E})$ for all $v \in\{1, \ldots, d\}$ and all $s \in[0,1]$. Fix $s \in[0,1]$, $v \in\{1, \ldots, d\}$, and let $s_{k} \underset{k \rightarrow \infty}{\longrightarrow} s$ where $s_{k}$ is a sequence of dyadic numbers. Now let

$$
F_{v, k}(\gamma):=x^{v}\left(\gamma\left(s_{k}\right)\right) \in Y \subset D_{Y}(\mathcal{E}), \quad k \in \mathbb{N} .
$$

Using Lemma 4.5.5 we have

$$
\begin{aligned}
\sum_{i=1}^{\infty} \lambda_{i}\left\langle S_{i}, D F(\gamma)\right\rangle_{\mathbb{H}}^{2} & =\sum_{i=1}^{\infty} \lambda_{i}\left\langle S_{i}(s), e_{v}\right\rangle_{\mathbb{R}^{d}}^{2} \leq \sup _{k} \sum_{i=1}^{\infty} \lambda_{i}\left\langle S_{i}\left(s_{k}\right), e_{v}\right\rangle_{\mathbb{R}^{d}}^{2} \\
& \leq \lambda_{v}+\frac{1}{2} \sum_{p=1}^{\infty} \frac{\lambda_{i_{p, v}}}{2^{p}} \leq \sum_{p=0}^{\infty} \frac{\lambda_{d 2^{p}}}{2^{p}}<\infty
\end{aligned}
$$

where $i_{p, v}$ is constructed as in Lemma 4.5.5. Furthermore $F_{v, k} \underset{k \rightarrow \infty}{\longrightarrow} F$ in $L^{2}(\varphi \nu)$ and

$$
\sum_{i=1}^{\infty} \lambda_{i}\left\langle S_{i}, D\left(F-F_{v, k}\right)(\gamma)\right\rangle_{\mathbb{H}}^{2} \leq \sum_{i=n_{k}}^{\infty} \lambda_{i}\left\langle S_{i}, D F(\gamma)\right\rangle_{\mathbb{H}}^{2}
$$

for some sequence $n_{k} \rightarrow \infty$ as $k \rightarrow \infty$. This says $F_{v, k} \underset{k \rightarrow \infty}{\longrightarrow} F$ in $\mathcal{E}_{1}$-norm and $F \in$ $D_{Y}(\mathcal{E})$, where we recall that $\|\cdot\|_{\mathcal{E}_{1}}^{2}=\mathcal{E}(\cdot, \cdot)+\|\cdot\|_{L^{2}(\varphi \nu)}^{2}$. Indeed, the same conclusion applies to an arbitrary $F \in Z$ with $F(\gamma)=f\left(\gamma\left(s_{1}\right), \ldots, \gamma\left(s_{k}\right)\right), s_{1}, \ldots, s_{k} \in[0,1]$ since because of (4.7) we have

$$
\left\langle S_{i}, D F(\gamma)\right\rangle_{\mathbb{H}}=\sum_{j=1}^{d} \sum_{i^{\prime}=1}^{k} \frac{\partial f}{\partial x_{i^{\prime}, j}}\left(\gamma\left(s_{1}\right), \ldots, \gamma\left(s_{k}\right)\right)\left\langle S_{i}\left(s_{i^{\prime}}\right), e_{j}\right\rangle_{\mathbb{R}^{d}}
$$

We then apply (4.9) and (4.17).

(c) Recalling (b), we still have to show that $Z \subset D_{Y}(\mathcal{E})$ implies (4.15).

We suppose $Z \subset D_{Y}(\mathcal{E})$. Therefore $x_{v}(\gamma(s)) \in D_{Y}(\mathcal{E})$ for all $v \in\{1, \ldots, d\}$ and all $s \in[0,1]$. First we check the expression of Lemma 4.5 .5 for all dyadic points $s_{k}$. To get a bound on

$$
\sum_{i=1}^{\infty} \lambda_{i}\left\langle S_{i}(s), e_{j}\right\rangle_{\mathbb{R}^{d}}^{2}
$$

when $s \in[0,1]$ is no longer dyadic, we need (4.15). The statement follows. 
Proposition 4.5.7. Let $\varphi$ satisfy (4.12). The form $\left(\mathcal{E}, D_{Z}(\mathcal{E})\right)$ is a Dirichlet form on $L^{2}(\varphi \nu)$.

Proof: We use Proposition I.4.10 from [5]. It follows that we must show $\mathcal{E}\left(1 \wedge F^{+}, 1 \wedge\right.$ $\left.F^{+}\right) \leq \mathcal{E}(F, F)$. We know that for $F \in Y$

$$
\begin{aligned}
\mathcal{E}(F, F) & =\sum_{i=1}^{\infty} \lambda_{i} \int\left\langle S_{i}, D F\right\rangle_{\mathbb{H}}^{2} \varphi d \nu=\sum_{i=1}^{\infty} \lambda_{i} \int\left(\partial_{S_{i}}, F\right)^{2} \varphi d \nu \\
& =\sum_{i=1}^{\infty} \lambda_{i} \int\left(\left.\frac{d}{d t}\right|_{t=0} F\left(\gamma+t S_{i}\right)\right)^{2} \varphi d \nu .
\end{aligned}
$$

Let $\xi_{\varepsilon}: \mathbb{R} \rightarrow[-\varepsilon, 1+\varepsilon]$ be non-decreasing such that $\xi_{\varepsilon}(t)=t$ for all $t \in[0,1]$, $0 \leq \xi_{\varepsilon}^{\prime} \leq 1$. It follows that $\xi_{\varepsilon} \circ F \rightarrow 1 \wedge F^{+}$. An application of the chain rule gives

$$
\begin{aligned}
\mathcal{E}\left(\xi_{\varepsilon} \circ F, \xi_{\varepsilon} \circ F\right) & =\int\left(\left.\frac{d}{d t}\right|_{t=0} \xi_{\varepsilon} \circ F(\gamma+t S)\right)^{2} \varphi d \nu \\
& =\int \xi_{\varepsilon}^{\prime}(F(\gamma)) \cdot\left(\left.\frac{d}{d t}\right|_{t=0} F(\gamma+t S)\right)^{2} \varphi d \nu \\
& \leq \int\left(\left.\frac{d}{d t}\right|_{t=0} 1 \cdot F(\gamma+t S)\right)^{2} \varphi d \nu \\
& =\mathcal{E}(F, F),
\end{aligned}
$$

from which we derive $\mathcal{E}\left(1 \wedge F^{+}, 1 \wedge F^{+}\right) \leq \mathcal{E}(F, F)$. Thus $\mathcal{E}$ is a Dirichlet form.

Proposition 4.5.8. Let $\varphi$ satisfy (4.12). The form $\left(\mathcal{E}, D_{Z}(\mathcal{E})\right)$ is local.

Proof: This is shown in the same manner as in Proposition 3.4 of [4] using the fact that $\nu$-a.e. implies $\varphi \nu$-a.e.

\subsection{Quasi-regularity}

It turns out that the closability condition found in the previous sections is sufficient for the form to be quasi-regular. Throughout this section we assume (4.12).

Proposition 4.6.1. Suppose (4.15). The form described by the closure of

$$
\mathcal{E}(F, F)=\sum_{i=1}^{\infty} \lambda_{i} \int\left\langle S_{i}, D F\right\rangle_{\mathbb{H}}^{2} \varphi d \nu, \quad F \in Z,
$$

in $L^{2}(\varphi \nu)$, is quasi-regular.

Proof: The conditions for closability can be found in Proposition 4.5.6. We follow the procedure of [4], see also [2, 9].

Step 1: For $r \in \mathbb{N}, l \in\left\{0, \ldots, 2^{r-1}-1\right\}$, and $k=2^{r-1}+l$, set $s_{k}:=(2 l+1) 2^{-r}$. Let 
$x^{v}(p)$ denote the $v$ th coordinate of $p \in \mathbb{R}^{d}, v \in\{1, \ldots, d\}$. Fix $\tau \in \Omega, k=2^{r-1}+l$, and $v \in\{1, \ldots, d\}$. Consider the functions $f_{v, k, \tau}(p):=x^{v}(p)-x^{v}\left(\tau\left(s_{k}\right)\right), p \in \mathbb{R}^{d}$, and

$$
F_{v, k, \tau}(\gamma):=f_{v, k, \tau}\left(\gamma\left(s_{k}\right)\right)=x^{v}\left(\gamma\left(s_{k}\right)\right)-x^{v}\left(\tau\left(s_{k}\right)\right), \quad \gamma \in \Omega
$$

clearly $F_{v, k, \tau} \in Y$. Using the procedure of Proposition 4.5.6(b) we get

$$
\mathcal{E}\left(F_{v, k, \tau}, F_{v, k, \tau}\right) \leq C_{1}<\infty
$$

where $C_{1}$ the bound obtained from (4.17).

Step 2: Let

$$
G_{n, \tau}=\sup _{\substack{k \in\{1, \ldots, n\} \\ v \in\{1, \ldots, d\}}}\left|F_{v, k, \tau}\right|, \quad n \in \mathbb{N},
$$

then

$$
\mathcal{E}\left(G_{n, \tau}, G_{n, \tau}\right) \leq \sup _{\substack{k \in\{1, \ldots, n\} \\ v \in\{1, \ldots, d\}}} \int \sum_{i=1}^{\infty} \lambda_{i}\left\langle S_{i}, D F_{v, k, \tau}(\gamma)\right\rangle_{\mathbb{H}}^{2} \varphi d \nu \leq C_{1}<\infty,
$$

as in [4] Lemma 3.2. We show $G_{n, \tau} \in L^{2}(\nu)$.

$$
\begin{aligned}
E\left[G_{n, \tau}^{2}\right] & \leq E\left[\left(\sup _{\substack{v \in\{1, \ldots, d\} \\
s \in[0,1]}}\left|x^{v}(\gamma(s))-x^{v}(\tau(s))\right|\right)^{2}\right] \\
& \leq 4 E\left[\sup _{\substack{v \in\{1, \ldots, d\} \\
s \in[0,1]}}\left(x^{v}(\gamma(s))\right)^{2}\right] \leq 4 E\left[\sum_{v=1}^{d} \sup _{s \in[0,1]}\left(x^{v}(\gamma(s))\right)^{2}\right] \\
& =4 d E\left[\sup _{s \in[0,1]}\left(x^{1}(\gamma(s))\right)^{2}\right] \leq 16 d=: C_{2}<\infty,
\end{aligned}
$$

where the last line is obtained using Doob's inequality. Thus

$$
\mathcal{E}_{1}\left(G_{n, \tau}, G_{n, \tau}\right) \leq C_{1}+C_{2}=: C_{3}<\infty .
$$

Step 3: Using (4.19) and the fact that the sequence $\left(G_{n, \tau}\right)_{n \in \mathbb{N}}$ satisfies $G_{n, \tau} \leq G_{n+1, \tau}$, $n \in \mathbb{N}$. By using Lemma I.2.12 of [5] we get that the function

$$
H_{\tau}(\gamma):=\sup _{\substack{s \in[0,1] \\ v \in\{1, \ldots, d\}}}\left|x^{v}(\gamma(s))-x^{v}(\tau(s))\right|, \quad \gamma \in \Omega,
$$

belongs to $D(\mathcal{E})$ and that

$$
\mathcal{E}_{1}\left(H_{\tau}, H_{\tau}\right) \leq C_{3}
$$

Let $\left\{\tau_{k}: k \in \mathbb{N}\right\}$ be a dense set in $\Omega$. Set

$$
K_{n}:=\inf _{1 \leq k \leq n} H_{\tau_{k}}, \quad n \in \mathbb{N} .
$$


Again using Lemma I.2.12 of [5] we have $K_{n} \in D(\mathcal{E})$ and $K_{n}$ bounded in $\left(D(\mathcal{E}), \mathcal{E}_{1}^{1 / 2}\right)$ from (4.20). We apply the Banach-Saks theorem, which states that every bounded sequence in $\left(D(\mathcal{E}), \varepsilon_{1}^{1 / 2}\right)$, has a subsequence whose Cesaro means converge strongly and we get $\mathcal{E}_{1}\left(K_{n}, K_{n}\right) \underset{n \rightarrow \infty}{\longrightarrow} 0$ since $\left\{\tau_{k}\right\}$ is dense. As $K_{n}$ is continuous, we may use Proposition 3.5 of [5] Chapter III, from which it follows that there exists a subsequence $K_{n_{k}}$, $k \in \mathbb{N}$, and an $\mathcal{E}$-nest $F_{m}, m \in \mathbb{N}$, such that $K_{n_{k}}$ converges uniformly to zero on each $F_{m}$ as $k \rightarrow \infty$. Let us follow an idea of [9], proof of Proposition 3.1. Given $\delta>0$ we can find $k$ such that $K_{n_{k}}<\delta$. We have

$$
F_{m} \subset \bigcup_{i=1}^{n_{k}} B\left(\tau_{i}, \delta\right)
$$

by (4.21), where $B(x, \delta)$ denotes the ball of radius $\delta$ centered at $x$. Now it follows that each $F_{m}$ is totally bounded. $F_{m}$ closed and totally bounded implies $F_{m}$ compact. Thus $F_{m}, m \in \mathbb{N}$ forms an $\mathcal{E}$-nest consisting of compact sets.

Step 4: For fixed $\tau \in \Omega$, the system of functions $F_{v, k, \tau}, v \in\{1, \ldots, d\}, k \in \mathbb{N}$ separates the points in $\Omega$, Step 3 showed that there is an $\mathcal{E}$-nest consisting of compact sets and the form is a Dirichlet form by Proposition 4.5.8. Quasi-regularity now follows from its definition.

Remark 4.6.2. We observe that to obtain (4.18) we need the condition (4.15). We recall that by Proposition 4.5.6(b) we have $\left(\mathcal{E}, D_{Y}(\mathcal{E})\right)=\left(\mathcal{E}, D_{Z}(\mathcal{E})\right)$.

Corollary 4.6.3. There exists a diffusion process $M$ associated with $\left(\mathcal{E}, D_{Z}(\mathcal{E})\right)$.

Proof: The result is an immediate consequence of Propositions 4.5.8 and 4.6.1 using Theorem IV.3.5 of [5].

\subsection{The generator}

Let $\varphi(\gamma)$ have the form of (4.6) and $\left(\mathcal{E}, D_{Y}(\mathcal{E})\right)$ given by

$$
\mathcal{E}(F, G)=\int\langle D F, A D G\rangle \varphi d \nu=\sum_{i=1}^{\infty} \lambda_{i} \int \partial_{S_{i}} F \partial_{S_{i}} G \varphi d \nu, \quad F, G \in Y
$$

where $D_{Y}(\mathcal{E})$ is the closure of $(\mathcal{E}, Y)$ on $L^{2}(\varphi \nu)$. We determine the generator $\mathbf{A}$ of this form, i.e.

$$
\mathcal{E}(F, G)=\int(-\mathbf{A} F) G \varphi d \nu, \quad F \in D(\mathbf{A}), G \in D_{Y}(\mathcal{E}) .
$$

Using $g_{n}$ from (4.8), let $\mathbf{S}_{i}(\gamma):=\int_{0}^{1}\left\langle g_{i}(s), d \gamma_{s}\right\rangle_{\mathbb{R}^{d}}, i \in \mathbb{N}$. We observe that $\mathbf{S}_{1}(\gamma), \mathbf{S}_{2}(\gamma), \ldots$ are independent $N(0,1)$.

Proposition 4.7.1. We have $Y \subset D(\mathbf{A})$. If $F \in Y$ then

$$
\mathbf{A} F=\sum_{i=1}^{\infty} \lambda_{i}\left[\partial_{S_{i}}^{2} F+\frac{\partial_{S_{i}} \varphi \partial_{S_{i}} F}{\varphi}+\mathbf{S}_{i} \partial_{S_{i}} F\right]
$$


Proof: Let $F, G \in Y$. Once again we note that the following sum is just a finite sum because of the particular structure of $Y$. We have

$$
\begin{aligned}
\mathcal{E}(F, G)= & \sum_{i=1}^{\infty} \lambda_{i} \int \partial_{S_{i}} F \partial_{S_{i}} G \varphi d \nu \\
= & \lim _{s \rightarrow 0} \sum_{i=1}^{\infty} \lambda_{i} \frac{1}{s}\left[\int G\left(\gamma+s S_{i}\right) \partial_{S_{i}} F \varphi d \nu-\int G \partial_{S_{i}} F \varphi d \nu\right] \\
= & \lim _{s \rightarrow 0} \sum_{i=1}^{\infty} \lambda_{i} \frac{1}{s}\left[\int G \partial_{S_{i}} F\left(\gamma-s S_{i}\right) \varphi\left(\gamma-s S_{i}\right) d \nu\left(\gamma+s S_{i}\right)-\int G \partial_{S_{i}} F \varphi d \nu\right] \\
= & \lim _{s \rightarrow 0} \sum_{i=1}^{\infty} \lambda_{i}\left[\int G \varphi\left(\gamma-s S_{i}\right) \frac{d \nu \circ T_{s S_{i}}}{d \nu} \cdot\left(\frac{\partial_{S_{i}} F\left(\gamma-s S_{i}\right)-\partial_{S_{i}} F}{s}\right) d \nu\right. \\
& \left.\left.+\int G \frac{d \nu \circ T_{s S_{i}} \partial_{S_{i}} F \cdot\left(\frac{\varphi\left(\gamma-s S_{i}\right)-\varphi}{d \nu}\right) d \nu}{s}\right) d \nu\right] \\
& +\sum_{i=1}^{\infty} \lambda_{i} \int-G \varphi \partial_{S_{i}}^{2} F-G \partial_{S_{i}} F \partial_{S_{i}} \varphi+\left.G \varphi \partial_{S_{i}} F \frac{d}{d s}\right|_{s=0} \frac{d \nu \circ T_{s S_{i}}}{d \nu} d \nu
\end{aligned}
$$

where $T$ is the shift operator, $T_{s S_{i}}(\gamma)=\gamma+s S_{i}$. Thus the generator satisfies

$$
\mathbf{A} F=\sum_{i=1}^{\infty} \lambda_{i}\left[\partial_{S_{i}}^{2} F+\frac{\partial_{S_{i}} \varphi \partial_{S_{i}} F}{\varphi}-\left.\partial_{S_{i}} F \frac{d}{d s}\right|_{s=0} \frac{d \nu \circ T_{s S_{i}}}{d \nu}\right]
$$

from which we can conclude $F \in D(\mathbf{A})$. The Cameron-Martin formula gives

$$
\left.\frac{d}{d s}\right|_{s=0} \frac{d \nu \circ T_{s S_{i}}}{d \nu}(\gamma)=-\mathbf{S}_{i}(\gamma)
$$

and (4.22) follows immediately.

Remark 4.7.2. Let $b_{j}$ denote $\left\langle b, e_{j}\right\rangle_{\mathbb{R}^{d}}$ and let $g_{i}$ be as in (4.8). The particular structure of $\varphi$ gives

$$
\begin{aligned}
\frac{\partial_{S_{i}} \varphi}{\varphi} & =\sum_{j=1}^{d} \int_{0}^{1}\left\langle D b_{j}\left(\gamma_{s}\right), S_{i}(s)\right\rangle_{\mathbb{H}} d\left(\gamma_{j}\right)_{s}+\int_{0}^{1}\left\langle b\left(\gamma_{s}\right), g_{i}(s)\right\rangle_{\mathbb{R}^{d}} d s \\
& -\frac{1}{2} \int_{0}^{1}\left\langle D\left\|b\left(\gamma_{s}\right)\right\|_{\mathbb{R}^{d}}^{2}, S_{i}\right\rangle_{\mathbb{H}} d s
\end{aligned}
$$


and therefore

$$
\begin{aligned}
\mathbf{A} F & =\sum_{i=1}^{\infty} \lambda_{i}\left[\partial_{S_{i}}^{2} F+\mathbf{S}_{i} \partial_{S_{i}} F+\left(\sum_{j=1}^{d} \int_{0}^{1}\left\langle\nabla b_{j}\left(\gamma_{s}\right), S_{i}(s)\right\rangle_{\mathbb{R}^{d}} d\left(\gamma_{j}\right)_{s}\right.\right. \\
& \left.\left.+\int_{0}^{1}\left\langle b\left(\gamma_{s}\right), g_{i}(s)\right\rangle_{\mathbb{R}^{d}} d s-\int_{0}^{1}\left\langle\sum_{j=1}^{d} b_{j}\left(\gamma_{s}\right) \nabla b_{j}\left(\gamma_{s}\right), S_{i}(s)\right\rangle_{\mathbb{R}^{d}} d s\right) \partial_{S_{i}} F\right] .
\end{aligned}
$$

\subsection{Local first moment}

Let $M=\left(\left(X_{t}\right)_{t \in[0,1]},\left(P_{\gamma}\right)_{\gamma \in \Omega}\right)$ be the corresponding diffusion process defined in Corollary 4.6.3, $S_{i}^{v}$ denote $\left\langle S_{i}, e_{v}\right\rangle_{\mathbb{R}^{d}}$, and $\mathbf{S}_{i}(\gamma)$ be as in Chapter 4.7. We recall that $(\mathbf{A}, D(\mathbf{A}))$ is the closure of $(\mathbf{A}, Y)$ in the graph norm $\left(\|\cdot\|_{L^{2}(\varphi \nu)}^{2}+\|\mathbf{A} \cdot\|_{L^{2}(\varphi \nu)}^{2}\right)^{1 / 2}$. Thus $\mathbf{S}_{i} \in$ $Y \subset D(\mathbf{A})$ by definition.

Proposition 4.8.1. We have

$$
\lim _{t \rightarrow 0} \frac{1}{t} \int\left(\mathbf{S}_{i}(\gamma)-\mathbf{S}_{i}(\tau)\right) P_{\tau}\left(X_{t} \in d \gamma\right)=\lambda_{i}\left[\frac{\partial_{S_{i}} \varphi(\tau)}{\varphi(\tau)}+\mathbf{S}_{i}(\tau)\right]
$$

Proof: According to Proposition (4.22), we have $\mathbf{S}_{i}(\gamma) \in Y \subset D(\mathbf{A})$. Therefore

$$
\lim _{t \rightarrow 0} \frac{1}{t} \int\left(\mathbf{S}_{i}(\gamma)-\mathbf{S}_{i}(\tau)\right) P_{\tau}\left(X_{t} \in d \gamma\right)=\mathbf{A}\left(\mathbf{S}_{i}(\tau)\right)
$$

and by (4.22)

$$
\begin{aligned}
\mathbf{A}\left(\mathbf{S}_{i}(\tau)\right) & =\sum_{j=1}^{\infty} \lambda_{j}\left[\partial_{S_{j}}^{2} \mathbf{S}_{i}(\tau)+\frac{\partial_{S_{j}} \varphi \partial_{S_{j}} \mathbf{S}_{i}(\tau)}{\varphi}+\mathbf{S}_{j}(\tau) \partial_{S_{j}} \mathbf{S}_{i}(\tau)\right] \\
& =\lambda_{i}\left[\frac{\partial_{S_{i}} \varphi(\tau)}{\varphi(\tau)}+\mathbf{S}_{i}(\tau)\right]
\end{aligned}
$$

For a dyadic number, $s_{k}=\sum_{i=1}^{r} c_{i} \cdot 2^{-i}$, we note that there exist $N\left(s_{k}\right) \in \mathbb{N}$ such that $\left\langle\left(s_{k} \wedge \cdot\right), S_{i}\right\rangle_{\mathbb{H}}=0$ whenever $i>N\left(s_{k}\right)$.

Proposition 4.8.2. (a) Let $s_{k} \in[0,1]$ be a dyadic number. Then $x^{v}\left(\gamma\left(s_{k}\right)\right) \in D(\mathbf{A})$ and

$$
\begin{aligned}
\mathbf{A} x^{v}\left(\tau\left(s_{k}\right)\right) & =\sum_{i=1}^{N\left(s_{k}\right)} \lambda_{i} S_{i}^{v}(s)\left[\frac{\partial_{S_{i}} \varphi(\tau)}{\varphi(\tau)}+\mathbf{S}_{i}(\tau)\right] \\
& =\lim _{t \rightarrow 0} \frac{1}{t} \int\left(x^{v}\left(\gamma\left(s_{k}\right)\right)-x^{v}\left(\tau\left(s_{k}\right)\right)\right) P_{\tau}\left(X_{t} \in d \gamma\right) .
\end{aligned}
$$


(b) Let $v \in\{1, \ldots, d\}$. Suppose (4.15) and

$$
\sum_{p=0}^{\infty} \frac{\lambda_{d 2^{p}}^{2}}{2^{p}}<\infty
$$

Then $x^{v}(\gamma(s)) \in D(\mathbf{A})$ and

$$
\begin{aligned}
\mathbf{A} x^{v}\left(\tau\left(s_{k}\right)\right) & =\sum_{i=1}^{\infty} \lambda_{i} S_{i}^{v}(s)\left[\frac{\partial_{S_{i}} \varphi(\tau)}{\varphi(\tau)}+\mathbf{S}_{i}(\tau)\right] \\
& =\lim _{t \rightarrow 0} \frac{1}{t} \int\left(x^{v}\left(\gamma\left(s_{k}\right)\right)-x^{v}\left(\tau\left(s_{k}\right)\right)\right) P_{\tau}\left(X_{t} \in d \gamma\right) .
\end{aligned}
$$

Proof: (a) We note

$$
\begin{aligned}
x^{v}\left(\gamma\left(s_{k}\right)\right) & =\int_{0}^{1} \chi_{\left[0, s_{k}\right]}(u) d \gamma_{u}^{v}=\int_{0}^{1} \sum_{i=1}^{\infty}\left\langle\chi_{\left[0, s_{k}\right]}, H_{i}\right\rangle_{L^{2}} H_{i}(u) d \gamma_{u}^{v} \\
& =\sum_{i=1}^{\infty}\left\langle\chi_{\left[0, s_{k}\right]}, H_{i}\right\rangle_{L^{2}} \int_{0}^{1} H_{i}(u) d \gamma_{u}^{v}=\sum_{i=1}^{\infty}\left\langle\left(s_{k} \wedge \cdot\right) e_{v}, S_{i}\right\rangle_{\mathbb{H}} \mathbf{S}_{i}(\gamma) .
\end{aligned}
$$

Since $s_{k}$ is dyadic the sum is finite and we get

$$
x^{v}\left(\gamma\left(s_{k}\right)\right)=\sum_{i=1}^{N\left(s_{k}\right)}\left\langle\left(s_{k} \wedge \cdot\right) e_{v}, S_{i}\right\rangle_{\mathbb{H}} \mathbf{S}_{i}(\gamma)=\sum_{i=1}^{N\left(s_{k}\right)} S_{i}^{v}\left(s_{k}\right) \mathbf{S}_{i}(\gamma) .
$$

(a) follows immediately.

(b) As in (a) we have $x^{v}(\gamma(s))=\sum_{i=1}^{\infty} S_{i}^{v}(s) \mathbf{S}_{i}(\gamma)$. Therefore $\sum_{i=1}^{\infty} \lambda_{i} S_{i}^{v}(s) \mathbf{S}_{i}$ converges in $L^{2}(\nu)$ and therefore also in $L^{2}(\varphi \nu)$ if $\sum_{i=1}^{\infty} \lambda_{i}^{2} S_{i}^{v}(s)^{2}<\infty$. We also have that $\sum_{i=1}^{\infty} \lambda_{i} S_{i}^{v}(s) \partial_{S_{i}} \varphi / \varphi$ converges in $L^{2}(\varphi \nu)$ if $\sum_{i=1}^{\infty} \lambda_{i} S_{i}^{v}(s)<\infty$. Using Lemma 4.5.5 and (4.23) the result follows.

Remark 4.8.3. For $f, g \in Y$, or $f, g \in Z$ if (4.15) holds, we have for $g \in D(\mathcal{E}) \cap L^{\infty}$, where $D(\mathcal{E})$ denotes $D_{Y}(\mathcal{E})$ or $D_{Z}(\mathcal{E})$ respectively

$$
\begin{aligned}
\int g \Gamma(f, f) \varphi d \nu= & -\mathcal{E}\left(g, f^{2}\right)+2 \mathcal{E}(f g, f) \\
= & -\int \sum_{i=1}^{\infty} \lambda_{i}\left(\partial_{S_{i}} f^{2}\right)\left(\partial_{S_{i}} g\right) \varphi d \nu \\
& +2 \int \sum_{i=1}^{\infty} \lambda_{i}\left(\partial_{S_{i}}(f g)\right)\left(\partial_{S_{i}} f\right) \varphi d \nu \\
= & \int g\left(2 \sum_{i=1}^{\infty} \lambda_{i}\left(\partial_{S_{i}} f\right)^{2}\right) \varphi d \nu
\end{aligned}
$$


where $\Gamma$ denotes the carré du champ operator (see [1] p. 17). Thus for $f \in Y \cap L^{\infty}$, or $f \in Z \cap L^{\infty}$ if (4.15) holds, we have

$$
\Gamma(f, f)=2 \sum_{i=1}^{\infty} \lambda_{i}\left(\partial_{S_{i}} f\right)^{2} .
$$

Using Proposition 4.8.1 we obtain

$$
\lim _{t \rightarrow 0} \frac{1}{t} \int\left\|\mathbf{S}_{i}(\gamma)-\mathbf{S}_{i}(\tau)\right\|_{\mathbb{R}^{d}}^{2} P_{\tau}\left(X_{t} \in d \gamma\right)=2 \lambda_{i}
$$

which formally coincides with Proposition 5.1 in [4]. The same holds for linear combinations of $\mathbf{S}_{i}(\gamma)$ belonging to $D(\mathcal{E})$. 



\section{Chapter 5-Notation and basic definitions}

\subsection{Introduction}

This chapter contains a selection of definitions and theorems that the reader is assumed to be familiar with from earlier experience. The reader could also read this section to refresh their memory. The section also has a list of notation used throughout the thesis.

\subsection{Definitions}

Definition 5.2.1. Given a measurable space $(X, \Sigma)$ and a measure $\mu$ on that space, a set $A$ is called an atom if

$$
\mu(A)>0
$$

and for any measurable set $B \subset A$

$$
\mu(B)=0
$$

If the measure $\mu$ has no atoms it is called non-atomic or atomless.

Definition 5.2.2. A Gaussian random variable $X$ is centered if

$$
E[X]=0
$$

Definition 5.2.3. A topological space $X$ is called separable if it contains a countable dense subset $Y$. In other words there exists a sequence $\left\{y_{n}\right\}_{n=1}^{\infty}$ in $Y$ such that every nonempty open subset of $X$ contains at least one element of this sequence.

Definition 5.2.4. A probability space $(\Omega, \mathcal{F}, P)$ is complete if for all $B \in \mathcal{F}$ such that $P(B)=0$ one has that for every $A \subset B$ that $A \in \mathcal{F}$. 
Definition 5.2.5. Let $X, Y$ be Banach spaces. A linear operator $A: \mathcal{D} \subset X \rightarrow Y$ is called closed if for every sequence $\left(x_{n}\right) \in \mathcal{D}(A)$ s.t. $x_{n} \rightarrow x \in X$ and $A x_{n} \rightarrow y \in Y$ one has $x \in \mathcal{D}(A)$ and $A x=y$.

Definition 5.2.6. Let $X, Y$ be Banach spaces. A linear operator $A: \mathcal{D} \subset X \rightarrow Y$ is called closable if for every sequence $\left(x_{n}\right) \in \mathcal{D}(A)$ s.t. $x_{n} \rightarrow 0$ and $A x_{n} \rightarrow y$, one has $y=0$.

Definition 5.2.7. A set of functions $\left\{f_{\alpha}: \alpha \in A\right\}: X \rightarrow \mathbb{R}$ is called a separating set for $X$ or said to separate the points of $X$ if for any two distinct elements $x$ and $y$ in $X$ there exists a function $f \in\left\{f_{\alpha}: \alpha \in A\right\}$ such that $f(x) \neq f(y)$.

Theorem 5.2.8. (Banach-Saks theorem) Let $H$ be a Hilbert space and $x_{n} \in H, n \in \mathbb{N}$ be bounded sequence, i.e. $\left\|x_{n}\right\| \leq C$ for all $n \in \mathbb{N}$. Then there exists a subsequence and an element $x \in H$ such that

$$
\frac{1}{N} \sum_{k=1}^{N} x_{n_{k}} \rightarrow x, \quad \text { as } N \rightarrow \infty .
$$

\subsection{Notation}

The same symbol can be used for different purposes. The principal notation is listed below, any deviations from this is explained in the text.

\section{Symbols and Operators}

$\begin{array}{ll}\langle\cdot, \cdot\rangle_{\mathbb{H}} & \text { The inner product in } \mathbb{H} \\ \|\cdot\|_{\mathbb{H}} & \text { The norm in } \mathbb{H} \\ \cdot \wedge \circ & \text { Minimum of } \cdot, \circ \\ \text { A } & \text { The generator } \\ \mathbb{1}_{A} & \text { The characteristic function of the set } A \\ \mathcal{B} & \text { The Borel } \sigma \text {-algebra } \\ C_{p}^{\infty} & \text { Smooth functions with polynomial growth } \\ D & \text { The Malliavin gradient operator } \\ D_{h} & \text { Directional derivative } \\ \mathbb{D}^{1, p} & \left.\text { Sobolev space (we have } D F \in L^{p}\right) \\ \delta & \text { The divergence operator } \\ \text { Dom } & \text { The domain } \\ D(\cdot) & \text { The domain } \\ E[\cdot] & \text { Expected value } \\ e_{i} & \text { Element of an ON-basis } \\ \mathcal{E} & \text { A bilinear form } \\ \mathcal{E}_{n} & \text { The set of elementary functions } \\ \mathcal{F} & \text { A } \sigma \text {-algebra }\end{array}$




$\begin{array}{ll}G_{\alpha} & \text { An element in the resolvent } \\ \mathbb{H} & \text { A Hilbert space. Often } L^{2}([0,1]) \\ H_{n} & \text { The } n \text {th Hermite polynomial, the } n \text {th Haar function } \\ \mathcal{H}_{n} & \text { The } n \text {th Wiener chaos } \\ I_{n} & \text { Stochastic integral of order } n \\ J_{n} & \text { The projection operator onto the } n \text {th Wiener chaos } \\ \Lambda & \text { The set of finite multiindices } \\ L & \text { The generator of the Ornstein-Uhlenbeck semigroup } \\ L_{s} & \text { Set of symmetric functions } \\ \mathbb{N} & \text { The natural numbers }\{0,1, \ldots\} \\ \Omega & \text { A sample space or the space } C_{0}\left([0,1) ; \mathbf{R}^{d}\right) \\ \Phi & \text { Density of a normal distribution } \\ \Phi_{a} & \text { A RV defined through Hermite polynomials } \\ \rho(\cdot) & \text { The resolvent set } \\ \mathbf{R} & \text { The real numbers } \\ \Gamma & \text { The carré du champ operator } \\ S_{i} & \text { The } i \text { th Schauder function } \\ \mathbf{S}_{i} & \text { Random variable linked to } S_{i} \\ T_{t} & \text { An element of the semigroup } \\ \mu & \text { A measure, often Lebesgue measure } \\ \nu & \text { A measure, often Wiener measure } \\ V[\cdot] & \text { The variance } \\ W_{t} & \text { Wiener process at time } t \\ \omega & \text { An element of } \Omega \\ Y & \text { A set of cylinder functions defined on dyadic points } \\ Z & \text { A set of cylinder functions } \\ Z_{\mathbb{H}} & \text { Cylinder functions with values in } \mathbb{H} \\ & \end{array}$

\section{Abbreviations and Acronyms}

$\begin{array}{ll}\text { a.e. } & \text { Almost everywhere } \\ \text { a.s. } & \text { Almost surely } \\ \text { i.i.d. } & \text { Independent and identically distributed } \\ \text { ON } & \text { Orthonormal } \\ \text { RV } & \text { Random variable } \\ \text { s.t. } & \text { Such that } \\ \text { w.l.o.g. } & \text { Without loss of generality } \\ \text { w.r.t. } & \text { With respect to }\end{array}$




\section{Bibliography}

[1] N. Bouleau and F. Hirsch. Dirichlet Forms and Analysis on Wiener Space. De Gruyter, 1991.

[2] B. Driver and M. Röckner. Construction of diffusions on path and loop spaces of compact riemannian manifolds. C. R. Acad. Sci. Paris, 315:603-608, 1992.

[3] M. Fukushima, Y. Oshima, and M. Takeda. Dirichlet Forms and Symmetric Markov Processes. De Gruyter, 2011.

[4] J.-U. Löbus. A class of processes on the path space over a compact riemannian manifold with unbounded diffusion. Trans. Amer. Math. Soc., 356, 2004.

[5] Z.-M. Ma and M. Röckner. Introduction to the Theory of (Non-symmetric) Dirichlet Forms. Springer, 1992.

[6] P.-A. Meyer. Stochastic processes from 1950 to the present. Electronic Journ@1 for History of Probability and Statistics, 5(1), June 2009.

[7] D. Nualart. The Malliavin Calculus and related topics. Springer, 2006.

[8] D. Nualart. The Malliavin Calculus and Its Applications. AMS, 2009.

[9] M. Röckner and B. Schmuland. Tightness of general $c_{1, p}$ capacities on banach space. J. Funct. Anal., 108:1-12, 1992.

[10] F.-Y. Wang. Weak poincaré inequalities on path spaces. Int. Math. Res. Not., pages 90-108, 2004.

[11] F.-Y. Wang and B. Wu. Quasi-regular dirichlet forms on riemannian path and loop spaces. Forum Math. Volume, 20(6):1085-1096, 2008. 
[12] F.-Y. Wang and B. Wu. Quasi-regular dirichlet forms on free riemannian path spaces. Infin. Dimens. Anal. Quantum Probab. Relat. Top., 12(2):251-267, 2009. 